\title{
IST KOENIG AELFRED IDER VERFASSER DER ALLITERIERENDEN UEBERTRAGUNG DER METRA DFS BOETIUS?
}

Bekanntlich kntipft sich die entstehung der literarischen angelsächsischen prosa an den namen könig Aelfred's. Mag auch die missionstätigkeit der angelsächsischen geistlichen ohne zweifel manche jetzt der vergessenheit anheimgefallene blite der beredsamkeit emporgetrieben haben, mag auch in den Witena Gemots einfluss und beredsamkeit von jeher hand in hand gegangen sein, so bleibt diesem könig doch das verdienst, der erste gewesen zu sein, der seinem vollse muster eines klaren, populären prosastils sichtbar vor augen führte. Wenn man die bedeutende ausdehnung seiner arbeiten in dieser richtung uberschaut - bedeutend auch nach dem sichtenden processe, welchem die moderne kritik den von alter zeit her uberlieferten katalog seiner schriften unterworfen hat -, so möchte es scheinen, dass der könig selbst sich seiner stärke als prosaschriftsteller bewusst war. Immerhin jedoch wuirde damit die möglichkeit eines gelegentlichen streifzuges auf das gebiet der poesie sehr wol vereinbar sein. Asser berichtet uns ja, dass Aelfred als knabe für die vaterländische dichtkunst sehr empfänglich war und manches lied auswendig wusste. Obne auf die mit seinem namen wirklich in verbindung gesetzten poetischen denkmäler bezug zu nehmen, liesse sich sehr wol denken, dass er sich diese begeisterung bis in das reife mannesalter erhalten habe, und dadurch angeregt, bier und da selbst als dichter aufgetreten sei.

So gibt es in der tat, von unsicheren fällen abgesehen, zwei unbestreitbare beispiele dieser art. Am schlusse seiner prosavorrede zur ubersetzung der Cura Pastoralis findet man 
einen alliterierenden prolng von 16 zeilen, und den schluss des gesammten werkes bildet ein ebenfalls in stabreimen geschriebener epilog von 30 zeilen. Allerdings muss man nun zugeben, dass eine eigentliche dichternatur uns aus diesen versen nicht entgegentritt. Das poetische darin beschränkt sich auf das alliterierende versmaass, und auch dies fällt durchaus nicht unter den klassischen typus. Der herausgeber des 'Hirdeboc' ist daher nicht übertrieben streng, wenn er die fraglichen verse als 'doggerel lines' charakterisiert.'

Auffallender muss es freilich auf den ersten blick erscheinen, des königs namen an der spitze eines so umfanglichen rerkes $\mathrm{zu}$ finden, wie die alliterierende übertragung der Metra des Boetius. Wird ihm dieselbe mit recht zugeschrieben, oder gehört sie in eine spätere zeit? Diese frage soll auf den folgenden seiten behandelt werden.

Was erstlich die äussere überlieferung der Metra anbetrifft, so ist darüber folgendes zu bemerken. Die alte pergamenthandschrift, die man als Cott. Otho A. 6 zu bezeichnen pflegt, und die nach Wanley 'vivente Alfredo aut saltem paulo post' geschrieben ist ${ }^{2}$, enthält die Aelfred'sche bearbeitung von Boetius' work mit der bearbeitung der Metra in stabreim, während die Bodleianische handschrift NE, C 3,11, welche nach Wanley's angabe aus dem anfange des 12. jahrhunderts stammt, die Metra nur in prosaischer bearbeitung enthält. In der prosaischen vorrede, welche an der spitze des gesammten werkes in beiden handschriften steht, wird ansdrücklich könig Aelfred als verfasser der alliterierenden tibertragung bezeichnet.

Werfen wir jetzt einen blick auf dic thber unsere frage bisher zum ausdruck gelangten ansichten. In älterer zeit dachte niemand daran, das werk dem könige abzusprechen. So sagt Rawlinson ${ }^{3}$, der erste herausgeber, in seiner vorrede:

'neque mihi sane dubium quin utraque ista Boethianorum carminum versio ab eodem prodierit Aelfredo, prior cum adversis pressus sensum dumtaxat auctoris exprimere satis haberet, posterior autem, cum

1 Sweet, in seiner ausgabe von Aelfred's Pastoral Care, s. 473.

2 Wanl. Catal. etc. Oxon. 1705 s. 217 . Ueber die schicksale dięser hs. vgl. Fox's ausgabe von Aelfred's Boetius, London 1864, s. III-IV.

3 Boethii de consolatione philosophiae libros $\mathrm{V}$ anglo-saxonice redditos ab Aelfredo inclyto Anglo-Saxonum rege ad apographum Junianum expressos edidit Christoph. Raw linson, 0xon. 1698 (Forrede s. II). 
otium postea nactns de poetica parte poetice qualitercunque reddenda cogitaret'.

Und weiter bemerkt er über den charakter dieser ubertragung:

'De poeseos huius indole ac genio haud aliud in praesentia habeo quod dicaw, quam quod colorem qaendam habeat a vulgari ac soluto sermone insigniter discrepantem; quanquam ad v́wos Caedmonianum haudquaquam adsurgat'.

Hickes ${ }^{1}$ machte die wahrnehmung, dass der stil der alliterierenden metra oft nicht im geringsten vom gewöhnlichen prosastile abweiche, drückte aber keinerlei zweifel über die verfasserschaft des königs aus. Wanley² in seinem Catalogus scbliesst sich an Rawlinson an. Denn er widerholt die oben zuerst angezogenen worte desselben und führt sie mit folgender bemerkung ein:

'Utramque metrorum Latinorum versionem tuto Aelfredo regi posse adscribi arbitratur (scil. Rawlinson), cuius quidem rei difficultatem sic argutissime solvit',

und nun folgen die citierten worte. Sharon 'l'urner ${ }^{3}$ drückt sich über den stil der alliterierenden metra ähnlich aus wie Hickes, bestreitet aber deswegen ebenso wenig die verfasserschaft des königs. Das gleiche gilt von Conybeare ${ }^{4}$, der die grosse einfachheit der diction durch ein bewusstes streben seitens des königs zu erklären sucht, so klar als möglich zu schreiben. Cardale 5 bemerkt in der vorrede zu seiner ausgabe der Aelfred'schen prosaúbertragung des Boetius, in deren anhang er eines der metra mitteilt:

'The poetical versions of the metra were a subsequent work, undertaken, doubtless in times of greater tranquillity and leisure.'

Ebenso wenig hatte $\mathrm{F}_{0 \times}{ }^{6}$, der zweite herausgeber der metrischen übertragung, irgend welche zweifel liber den verfasser:

1 Hickesii thesaurus ling. septent. Oxon. 1795, I s. 177-78.

2 A. 2. o. s. 85.

${ }^{3}$ Sh. 'l'urner, History of the Anglo-Saxons from the earliest times to the Norman conquest, 1799. Pariser ausgabe von 1840, II s. 65.

4 Illustralions of Anglo-Saxon poetry, ed. by Conybeare, London 1826 , s. $258-59$.

- King Alfred's Anglo-Saxon version of Boethius de Consolatione Philosophiae with an English translation and notes by Cardale. London 1529. (Jak. Grimm in seiner anzeige dieses werkes, Gütt. Gel. Anz. 1833, s. 1587 , bedauert, dass Cardale die Metra nicht mitgeteilt habe, die doch ein wichtiger teil von könig Aelfred's worke seien.).

- King Alfied's Anglo-Saxon version of the metres of Boethius with an English translation and notes by the Rev. S. Fox, Loudon 1835. 
'When the king had overcome the difficulties which beset him, he reduced the translation of the metres to that form in which they have been handed down to us, being at once a monument of royal industry and a pure specimen of the poetry of the Anglo-Saxons'. (!)

Edwin Guest ${ }^{1}$ betrachtet die verfasserschaft des königs als sicher, bemerkt aber zugleich:

'Alfred's versification shows poorly beside that of Caedmon. He seems to have had little more command over his rhythm than some of our modern poets'.

Auch Arend2, in seiner Geschichte der angelsächsischen Literatur, schliesst sich der hergebrachten meinung an:

'zeker is het, dat zij (i. e. de vertaling der Metra) eene cchte proeve van Angel-Saksische dichtkunst aanbiedt, en, gelijk trouwens de bewerking van den geheelen Boethius, een heerlijk gedenkteeken is van koninglijken ijver en onverpoosde vlijt. Zij kenmerkt overal den echt wijsgeerigen en verlichten geest, welke den edelen vorst bezielde'.

In dem nämlichen jahre aber, in welchem dies letzt erwähnte werk reröffentlicht wurde, erklärte sich Thomas Wright ${ }^{3}$ in entschiedener weiso gegen dic authenticität der angelsächsischen Metra. Dieser brachte im wesentlichen drei argumente dagegen vor: 1. der verfasser begeht den groben fehler, dass er drei metra zu versificicren unterlässt, weil sie in könig Aelfred's prosaubertragung nicht durch die gewöhnliche formel eingefthrt wurden; 2. die metra sind ausserordentlich schwach vom dichterischen standpunkte aus; 3. der verfasser hat eine höchst mangelhafte kenntniss des klassischen altertums und begeht fehler selbst da, wo Aelfred das richtige hat. Auf grund dieser drei argumente bestreitet Wright die verfasserschaft des königs. Nach ihm gehören die metra

Wideraufgelegt in Bohn's Antiq. Lib. London 1864 (Preface). Ein anonymer recensent von Fox's Boetius im Gentleman's Magazine, London 1835 , s. 49, sagt: Alfred's poetical version of the metres was a subsequent work. The introduction originally prefixed to the Cott. ms. and therefore properly given by Mr. Fox, is evidently not the production of Alfred himself. These lines are, however, an additional proof, if any were wanting, that our glorious king Alfred was the translator of Boethius and the author of the metrical version.

1 E. Guest, Hist. of Engl. Rhythms, London 1838, II, s. $50 \mathrm{ff}$.

2 J. P. Arend, Proeve eener geschiedenis der dichtlcunst en fraaije letteren onder de Angel-Saksen, Amsterdam 1842, s. 80.

3 Th. Wright, Biographia Britanica Literaria, Anglo-Sason-Period, Londun 1842, 8. 56-57, 400-403. 
irgend einem unbekannten dichterling des zehnten jahrhunderts an.

Seitdem sind die ansichten der gelehrten ther diese frage geteilt gewesen. Verschiedene traten Thomas Wright bei. So Pauli, der allerdings kein neues argument vorbringt. Ebenso Weiss'; dieser hat eine zu hohe meinung vom könige, als dass er ihn sich als verfasser der metra denken könnte. Obne zu bestreiten, dass die metra vom könige in versform umgesetzt worden sind, erklärt er doch nachdrücklich, dass diejenigen metra, welche uns erhalten sind, auf keinen fall von ihm herrihiren können. Aehnlich spricht sich Bouterwek ${ }^{3}$ aus. Aush G. Guizot ${ }^{4}$ bestreitet sehr entschieden die verfasserschaft des königs, widerholt aber im grunde nur die einwände Wright's. Auch Morley ${ }^{5}$ schliesst sich den genannten an, ohne inde'ss etwas neues vorbringen zu können. Wülcker ${ }^{6}$ erklärte sich gelegentlich für die Wright'sche ansicht. Rieger? hält die metra für das werk irgend eines späteren verfassers.

Andere erklären sich nicht bestimmt für oder gegen. So Ettmüller8, der sich darauf beschränkt zu sagen, dass die alliterierende bearbeitung der metra dem könig zugeschrieben wird. Auch Hammerich ${ }^{9}$ scheint über die frage nicht in's reine gekommen zu sein.

Andererseits fehlt es nicht an solchen, die trotz Wright Aelfred's ansprtiche auf das in rede stehende werk weiter vertreten haben. So M. Tupper ${ }^{10}$, verfasser einer gereimten neuenglischen ibersetzung der metra. Tupper verdient besondere

1 Pauli, Alfred der Grosse, Berlin 1851, s. 225-26.

2 Weiss, Geschichte Alfred's d. Gr., Schaffhausen 1852, s. 212.

3 Caedmon, herausg. von Bouterwek, Gütersloh 1854, pref. s. XLIX.

1 G. Guizot, Alfred le Grand, 2e éd. Paris 1863, 8. 205-206.

- Morley, English Writers, London 1867, I, s. 400.

6 Vgl. Paul und Braune's Beiträge z.g.d.d.sp. I, 1874, s. 241 anm.

7 Rieger, Alt- und Angelsächs. Verskunst, Halle 18i6, s. 32-35.

8 Ettmüller, Handbuch der Deutschen Literaturgeschichle etc., Leipzig 1947 , s. 145.

9 Hammerich, De episk-kristelige oldkvad hos de gotiske folk, Kjübenhavn 1873 , s. 90 .

${ }^{10}$ King Alfred's Poems now first turned into English Metres by M. F. Tupper, London 1850. Wider abgedruckt in der sogen. 'Jubilee Edition' von Aelfred's werken, London 1858, I, s. 158-219 und in Fox's ansgabe des Boetius, London 1864, s. 263-352. 
erwähnung als ein bewunderer des dichterischen wertes der metra, wobei es allerdings fraglich ist, ob er, unter dem einflusse einer art von selbsttäuschung stehend, nicht sowol das original als seine eigene ubertragung bewundert. In bezug auf die fragliche authenticität bemerkt er: 'It may be more learned to doubt, but it is far more sensible to believe'. Dietrich1 citiert widerholt. könig Aelfred als den verfasser der.metra, hat jedoch, wie es scheint, niemals die frage eingehender untersucht. Grein ${ }^{2}$ druckt die metra als Aelfred's werk ab. Die zehn einleitenden verse schreibt er einem andern verfasser zu, erhebt jedoch keinerlei zweifel darbiber, dass die metra selbst von Aelfred herrühren. Sweet ${ }^{3}$ sieht sie ebenfalls als Aelfredisch an, ohne indess. Wright's einwänden entgegenzutreten. Ten Brink ${ }^{4}$ endlich erklärt sich entschieden zu gunsten der authenticität. Wenngleich indess es nach seinen äusserungen nicht unwahrscheinlich ist, dass Wright's standpunkt aufzugeben ist, so bat er doch nicht alle schwierigkeiten aus dem wege geräumt. Ueber einen wichtigen punkt spricht er nur andeutungsweise, und ein argument des englischen kritikers, bei weitem nicht das geringste, berücksichtigt er uberhaupt gar nicht. Kurz, auch nach ten Brink kann die frage durchaus noch nicht als entschieden betrachtet werden. Dieselbe soll dalier in den folgenden seiten einer eingehenden untersuchung unterzogen werden.

In anbetracht des umstandes, dass alle späteren einwände und zweifel auf die Wright'sche kritik von 1842 zurlickgehen, und seitdem nichts wesentlich neues vorgebracht worden ist, wird der naturlichste weg der untersuchung der sein, Wright's argumente sorgfăltig nach einander zu prüfen. Nachdem ich dieselben auf ihren wabren wert zurtickgefuhrt habe, gedenke ich die authenticität der metra noch durch einige andere er-

1 Haupt's Zeitschrift f. d. A. 1853, s. 218-219. Anglo-Saxonica (universitätsprogramm von Marburg) 1854 , s. VI. Niedner's Zeitschrift für hist. Theol. 1856, s. 231.

2 Grein's Bibl. d. ags. Poesie etc. II. bd. 'Text II. Güttingen 1858, 8. $295-339$, s. $412-413$.

${ }^{3}$ Sweet, Characteristics of ags. poetry, in dem 2. bande der nenen ausgabe von Warton's History of Engl. Poetry. London 1871, s. 8.

4 ten Brink, Geschichte der englischen Literatur 1. bd. Berlin 1577, s. $100-102$. 
wägungen ausser zweifel zu stellen. Der bequemlichkeit halber bezeichne ich die lateinischen metra mit $A$, die angelsächsische prosalibertragung derselben mit $\mathrm{B}$, und die alliterierende ubertragung mit $\mathbf{C .}{ }^{1}$

Doch vor allem ist der hier und da auftretende irrtum zu beseitigen, dass $\mathrm{C}$ auf $\mathrm{A}$ beruhe, dass der angelsächs. dichter, wer immer es gewesen sein mag, den latein. text der metra vor sich gehabt hätte, als er seine zeilen schrieb. Auf grund einer derartigen anschauung sprechen Turner ${ }^{2}$, Conybeare ${ }^{3}$, 'I'upper 4 von der grossen freiheit, die der verfasser von $\mathbf{C}$ sich bei der bebandlung der lateinischen metra des Boetius gestattet hätte. Auch Grein ${ }^{5}$ scheint dieser anschauung gehuldigt zu haben. Wenigstens vergleicht er $\mathrm{C}$ mit $\mathrm{A}$ und findet dabei, dass neun lat. metra von $\mathrm{A}$ in $\mathrm{C}$ fehlen. Grein's ansicht hiertiber lässt sich nicht mit absoluter sicherheit feststellen, indess könnte jemand aus seinen worten sehr wol entnehmen, dass der verfasser von $\mathrm{C}$ bei seiner arbeit $\mathrm{A}$ vor sich hatte, und neun metra, sei es mit oder ohne absicht, ausliess. Dies wurde indess eine irrige vorstellung sein. Ich habe diese fiage eingehend gepruft und kann daher mit sicherheit behaupten, dass der verfasser von $C$ sich niemals die mule nahm, das lat. original aufzuschlagen, dass er ausschliesslich nach B arbeitete. ${ }^{6}$ Die ähnlichkeit zwischen $C$ und $B$ ist so schlagend, als möglich. Nicht nur folgt $C$, mit einigen sehr unbedeutenden ausnahmen, getreu dem gedankengange so wie er in B vorliegt,

1 Leider gibt es keine genane beschreibung der hs. Cott. Otho A. 6. Einige punkte wiirden vielleicht schon durch den charakter derselben zu entscheiden sein. Ob die ags. glossen in der lat. C. C. C. C. hs. des Boctius irgend welchen bezug zn Aelfred's werk haben, ist noch nicht untersucht. Wanley sagt a. a. o. s. 151: 'In eo habentur A. M. Boethii libri de Consol. Philos. Primus et pars libri secundi tam in textu quam in margine glossatur Saxonice'.

2 A. a. o. s. 66.

3 A. a. o. s. 259.

4 Jubilee Edition of King Alfred's Works, I, s. 179.

5 A. a. o. s. 413.

- Dies ist allerdings schon von Th. Wright bemerkt worden, a.a.o. s. 57, doch nicht mit dem nötigen nachdruck: "The metra are little more than a transposition of the words of Alfred's onn prose, with here and there a few additions and allerations to make allitcration'. Es war nötig, diesen punkt gleich aw eingange der untersuchung klar zu stellen. 
sondern es behält auch, so weit als dies überhaupt mit der natur der alliteration vereinbar ist, die worte und wendungen von $B$ bei. Wo immer sich in $C$ zusätze finden, die in der regel unbedeutend sind und meist einen rein ausfullenden charakter haben, sind sie niemals, in keinem einzigen beispiele, aus A entlehnt, ja sie sind nicht einmal indirekt durch A hervorgerufen. Nur wenige zeilen in $\mathrm{C}$ finden keinen entsprechenden text in B. Hier sei indess einer möglichen falschen auffassung vorgebeugt. Der ags. umdichter verfuhr nicht so mechanisch, dass er sich bei seiner arbeit ausschliesslich darauf beschränkte, B zu lesen. Wenn man aus einigen stellen einen schluss ziehen darf, so las er die ganze ags. prosaubertragung durch, ebe er sich an die alliterierende umdichtung der Metra machte. Diese stellen sind 26, ${ }_{87-88}$ :

swelcum he ôror

on his lîfdazum zelîcost wæs,

welche worte auf der vorhergehenden prosaübertragung beruhen, und $5,44-45$ :

pæt hit sêo êce ne môt innan geondscînan

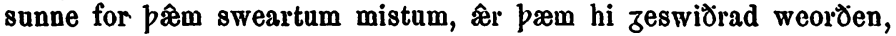

auch diese verse haben ihre quelle in den schlusssätzen der vorkergehenden prosa.

Nachdem so die wirkliche grundlage von C festgestellt ist, hat es durchaus nichts uberraschendes, wenn sechs verschiedene metra des Boetius, welche Aelfred in B ausliess - ich brauche hier nicht $\mathrm{zu}$ untersuchen aus welchen gründen -, wenn diese metra auch in C fehlen: $1,3 \cdot 4 \cdot 2,1.5,1 \cdot 3 \cdot 4$. Diese auslassungen sind ohne irgend welche bedeutung flir unsere frage.

Nach diesen vorbemerkungen untersuche ich zuerst den beweis Wright's, welchen ich als den gewiclitigsten betrachte: der umstand nämlich, dass der umdichter drei verschiedene metra $\left(\mathrm{I},{ }_{6} . \mathrm{II},{ }_{2} . \mathrm{IV},,_{7}\right)$, die ibren gebuhrenden platz in Aelfred's prosaübertragung hatten, vollständig ausgelassen hat. Nach Wright hätte der verfasser von $\mathrm{C}$ dieselben aus reiner nachlässigkeit ubersehen, weil er in der prosa Aelfred's an ihrer spitze nicht die formel fand, durch welche ein metrum in der regel eingeleitet wird.

Richtig ist allerdings, wie Wright sagt, dass Aelfred in seiner. prosa die iibersetzung eines metrums gewöhnlich durch eine gewisse formel einfubrt. Ja noch mehr! In den meisten 
fällen ist nicht nur eine derartige einleitende formel vorhanden, sondern der verfasser charakterisiert auch in der regel jedes metrum, unmittelbar nachdem er es gegeben hat, als 'leod', einmal als 'fytte', so dass dasselbe, von diesen zwei stehenden formeln so zu sagen eingerahnit, sehr leicht erkennbar wird. Die einleitende formel ist:

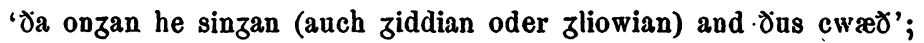
die schlussformel lautet:

' $\partial_{a}$ se Wisdom $\partial_{a} \partial_{i s}$ leoð asunzen hæfde, $\partial_{a}$ etc. Beide diese formeln werden bei den folgenden metren gebraucht:

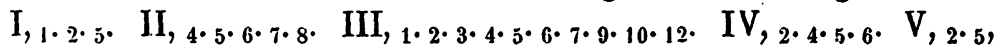
d. h. also in 24 fällen von 30.1 Nur die einleitende formel wird gebraucht: $\mathrm{II},_{3}$. III, $8 \cdot 11$. IV,,$_{1 \cdot 3}$. Weder die eine noch die andere kommt vor: I, ${ }_{6} \cdot 7 . \mathrm{II},{ }_{2}$. IV,,$^{2}{ }^{2}$

Wenn man nun bedenkt, dass Aelfred seinem lateinischen texte durchweg sehr frei gegenubersteht, so liesse sich sebr wol fragen, ob er iberhaupt die absicht hatte, diese vier metra in seine version als metra aufzunehmen, oder ob er es nicht lieber vorzog, aus irgend welchem grunde, sie nur seiner eigentlichen prosa einzuverleiben. Das weglassen beider formeln scheint fur diese auffassung zu sprechen. Indess ist dieselbe kaum anwendbar auf Metrum $I_{7}$. Ich will keinen besonderen wert darauf legen, dass sich die bearbeitung dieses metrums in ihrem ganzen tone von dem eigentlichen prosatexte abhebt und sich dadurch als metrum kenuzeichnet. Allerdings findet man an seiner spitze nicht die gewöhnliche formel, indess scheinen die unmittelbar vorhergehenden sätze sehr dafür zu sprechen, dass wir es hier mit einem metrum zu tun haben. Nachdem nämlich die philosophie zuror von dem nebel gesprochen, welcher das geistige auge verdunkelt, fährt sie fort:

'ac ic hie sceal ærest zeðinnian, خæt ic siððan خy eð mæze خæt soðe leoht on je zebrinzan',

was nur bedeuten kann: Ehe die philosophische belehrung, wie sie in der vorhergehenden prosa gegeben worden ist, in dem

1 Ich sage 30 , nicht 31 . Denn was man zuweilen das erste metrum nennt, ist weiter nichts als die historische cinltitung zu dem ganzen werke.

2 Es ist nicht korrekt, wenn Wright (1. c. s. 403) mit bezug auf die metra I,. II, ${ }_{2}$ IV, ${ }_{7}$ angibt: 'In these three instances only he has omitted that expression in the proseversion, which led the vriter of the metrical version to overlook them entirely'. 
nächsten buche mit vorteil fortgesetzt werden kann, muss der das geistige auge verdunkelnde nebel beseitigt werden. Dies geschieht durch das metrum.

Die metra haben im allgemeinen keinen philosophischen inhalt, sondern bilden gewissermassen ruhepunkte nach den abstrakten erörterungen der prosa. Andererseits wird das in frage stehende stück von Aelfred allerdings nicht als 'leod'' bezeichnet, doch folgt unmittelbar darauf eino wendung, welche sich ebenfalls mehr als ein mal nach der übertragung eines metrums findet:

'da zeswizode se Wisdom ane lytle hwile'.

Diese wendung wird, sei es in derselben form, sei es mit einer geringen änderung, nach den folgenden metris gebraucht, und zwar nur in diesen fällen: II, ${ }_{6}$. III, ${ }_{1}$. IV $_{4 \cdot 5} . \mathrm{V},{ }_{2}$. Dies scheint ziemlich deutlich darauf hin $\mathrm{zu}$ weisen, dass der könig selbst von vornherein diesen teil des textes als ein metrum angesehen wissen wollte.

Wie ist nun aber das nichtrorhandensein der drei anderen metra I, ${ }_{6}$ II, ${ }_{2} . \mathrm{IV},{ }_{7}$ in $\mathrm{C} \mathrm{zu}$ beurteilen? Zuerst entsteht hier die frage, ob diese auslassung nicht viel mehr auf reclınung des schreibers als auf die des verfassers von $\mathrm{C}$ zu setzen ist. Dass wir es hier nicht mit dem muster eines schreibers zu tun haben, gelit daraus hervor, dass er offenbar aus reiner nachlässigkeit den grössten teil des 23. metrums ausgelassen hat. Obgleich dies eine unzweifelhafte tatsache ist, so scheint sie doch noch niemandem aufgefallen zu sein. Denn die herausgeber der metra, Rawlinson, Fox und Grein, drucken das fragliche stlick, ohne irgendwie anzugeben, dass es ein fragment ist, und aus der ubertragung Tupper's sieht man, dass er es ebenfalls nicht bemerkt hat. Man braucht aber nur v. $7-9$ zu lesen:

We sculon pêah zita mid 3odes fylste ealdum and lêasum pînne inzeponc bêtan bîspellum etc.

und damit die entsprechenden worte der prosaübertragung zu vergleichen $(\mathrm{XXXV}, 6)$ :

'we sculon zet of ealdum leasum spellnm de sum bispell reccan'.

Diese worte bestehen nicht für sich selbst, sondern dienen nur dazu, die unmittelbar darauffolgende, geschichte von Orpheus und Eurydice einzuftiluren. Und am ende des ganzen finden sich die worte: 


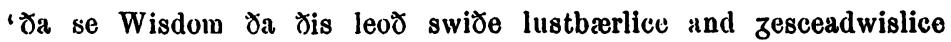
asunzen hrefde' ctc.

so dass über den charakter des vorausgehenden textes keinerlei zweifel bestehen kann. Da nun diese geschichte von Orpheus und Eurydice in $\mathrm{C}$ weggelassen ist', obgleich der umdichter dazu keineswegs durch die prosaïbertragung verleitet werden konnte, und obgleich er selbst sie deutlich genug eingeleitet hat, so kain die auslassung nur dem schreiber, nicht dem umdichter selbst zur last gelegt werden.

Nachdem so einmal diese nachlässigkeit des schreibers erwiesen worden ist, darf man ihm wol zutrauen, dass er im stande war, drei metra ganz und gar auszulassen? Diese möglichkeit ist nicht schlechterdings ausgeschlossen. Wenn man jedoch die oben festgestellte tatsache in betracht zieht, dass $\mathbf{C}$ ausschliesslich auf $B$ und nicht auf $A$ beruht, und dass die ïbertragungen der drei fraglichen metra das gemeinsame kennzeichen haben, dass sie sich durch keinerlei äussere bezeichnung von dem umstehenden texte abheben, so ist sehr wol denkbar, dass die auslassungen in $\mathrm{C}$ auf rechnung des verfassers selbst zu setzen sind. Es kann durchaus nicht eingeräumt werden, dass man aus der tatsache der auslassung ein argument gegen die verfasserschaft des königs herleitet. Wright meint, die auslassung in $\mathrm{C}$ sei ein grober schnitzer, der unmöglich von könige selbst begangen worden sein kann, sondern nur von einem mechanisch arbeitenden bearbeiter. Da er am eingange der beztiglichen abschnitte die gewöhnlicho formel nicht bemerkte, so kam er taberhaupt nicht auf den gedanken, dass in diesen fällen metra vorlägen, und liess sie daher ganz aus. Dies ist aber doch nicht eine ganz genaue darstellung des sachverhaltes. Obwol Wright sehr wol wusste, dass B die quelle von $\mathrm{C}$ ist, kam or durch die unbestreitbare tatsache der auslassung zu dem allzu raschen schlusse, dass die metra nicht von Aelfred herrihhren können. Wenn jemand zu tadeln ist, so ist es nicht der verfasser von $\mathrm{C}$, sondern der von B. Für jemanden, der nicht das lat. original zur hand hat, duirte es

1 Man sicht nicht recht, wie Turner (1. c. s. 65-66) sagen kann: 'The reader who compares the description of the golden age and the stories of Eurydice and Circe, inserled before from Alfred's prose with his translations of the same into verse, will perceive that his poetry has not increased their interest'. 
kaum müglich sein, die ïbertragungen der drei metra aus dem ubrigen angelsächs. texte herauszufinden, kaum möglich sein, den punkt anzugeben, wo sie anfangen. Und man kann daher nicht unverständiger weise zweifeln, ob Aelfred iberhaupt die absicht hatte, sie als eigentliche metra zu geben. Dies gilt besonders von metrum $I_{,}$. Denn dieser teil ist soigar mit der vorausgehenden prosa durch die partikel 'for ठan' direkt verknüpft. Es würde daher unbillig sein, dem verfasser von $\mathrm{C}$ deswegen einen vorwurf machen zu wollen. So wie er arbeitete, war es nicbt anders möglich, als dass er die drei metra ausliess. Es ist schlechterdings unzulässig, von dieser grundlage aus könig Aelfred's verfasserschaft anzufechten. Nachdem er einmal seine prosatubertragung des Boetius vollendet hatte - fuir ihn, unter den damaligen verhältuissen, wahrlich keine kleine aufgabe -, war es dann nicht ganz natiirlich, dass er sich nun nicht noch einmal die arbeit aufbürdcte, den text der lat. metra zu entziffern? Der metra, die ihm ohne zweifel als ein schweres lesestïck oft ziemlich rätselbaft erschienen sein müssen. Man bedenke nur, dass die dichtung des Boetius oft recht dunkel ist, wegen ihrer ausserordent lichen gedrängtheit und ihrer mannigfachen anspielungen mythologischer und anderer art, die für einen gelehrten des 9. jahrhunderts nicht selten ganz unverständlich sein mussten. Diese erwägungen lassen es ganz begreiflich erscheinen, dass Aelfred als grundlage für seine umdichtung nicht den lat. text, sondern seine eigene prosaubertragung nahm. Man darf annehmen, dass ein gewisser zeitraum, vielleicht von einigen jahren, zwischen den beiden arbeiten liegt. In der zwisènenzeit konnte ein geist wie der Aelfred's nicht unbeschäftigt bleiben. Und so ist es durchaus nicht auffällig, dass er bei wideraufnahme seiner arbeit behufs umdichtung der metra die drei fraglichen sticke ganz ubersah, die ja kein äusserliches kennzeichen trugen und so in dem texte gewissermassen begraben lagen.

Noch eine erörterung in bezug auf metrum IV, 7 . Unmittelbar nach der tubertragung derselben liest man die worte:

' $\gamma_{a}$ se Wisdom $\gamma_{a}$.

Die anwendung des wortes spell auf den vorhergehenden text spricht erstens dafür, dass könig Aelfred selbst bei abfassung seiner prosalubertragung diesen teil nicht als ein metrum angesehen wissen wollte, und zweitens musste sie den verfasser 
von $\mathrm{C}$ zu der annahme verleiten, dass es sich hier um einen teil der eigentlichen prosa handele. Denn nach dem allgemeinen sprachgebrauche der prosaïbertragung bedentet spell prosa, im gegensatze zu leo $\delta, z y d$, fytle, welche ausdrücke für metrum stehen. In diesem sinue wird spell oder spellian vor den folgenden metris gebraucht: II, $4 \cdot 5 \cdot 6 \cdot 7 \cdot 8 . \mathrm{III}, 1 \cdot 2 \cdot 3 \cdot 4 \cdot 5 \cdot 6 \cdot 7 \cdot 8$. $10 \cdot 11 . \mathrm{IV}, 1 \cdot 2 \cdot 3 \cdot 4 \cdot 5 \cdot 6 \cdot \mathrm{V}, 2 \cdot 5$, und $\mathrm{nach}$ folgenden metris: II, $4 \cdot 5 \cdot 7 \cdot$ III, $2 \cdot+\cdot 5 \cdot 6 \cdot 7 \cdot 9 . \mathrm{IV},{ }_{2} ;$ d. b. also in 33 verschiedenen fällen. Nur eine wirkliche ausnahme ${ }^{1}$ gibt os in Aelfred's Boetius von dieser

2 Eine anscheinende ausnahme findet sich im 2. verse des 7 . metrums:

'zlîowordum zôl zyd æt spelle'.

Hier scheinen die beiden ausdruicke unterschiedslos gebraucht zu sein. Indess stebt in der hs. nicht oel, sondern ast. Diese lesart gibt keinen sinn. Sie in oet zil andern ist nicht tunlich, denn wie sollte man sich den buchstaben vor dem $t$ erklären? Andrerseits ist Ettmüller's verbesserung: 'gyd ecle spelle' doch allzu gewaltsam. Die wirkliche emendation diirfte folgende sein: Statt aest ist zu lesen ceft. Bekanntlich ist nur ein geringer unterschied zwischen der ags. form von $f$ und $s$. Urspriinglich befand sich vielleicht iiber dem $f l$ ein kleiner strich, dic abkürzung für $e r$, der indess ans versehen weggelassen sein kann. So kommt man zu der befriedigenden lesart:

'zliowordum zôl zyd æefter spelle',

d. h. nach der vorhergehenden prosa begann die philosophie zu singen. Eine andere scheinbare ausnahme ist der gebrauch von spellian in dem unmittelbar auf das 14. metrum folgenden satze:

' $p \hat{a}$ se Wîsdôm pầ Jis lîot âsunzen hæfde, pâ onzan he eft spellian and cwæó'.

Aus dem doppelpunkte, welchen Grein nach diesem satze setzt, sieht mall, dass er eine irrige anschauung von den ags. metren hat. Denn nach dieser interpunktion wïrde der in rede stehende eatz uatürlich das folgende metrum einführen, so dass also ein metrum auf das andere folgen würde. Doch zeigt ein blick auf die oben citierten sätze hinlïnglich, wie der satz zu verstehen ist. Er kündigt nämlich einfach die auf das metrum folgende prosa an, und musste daher besser ganz ausgelassen werden, wenn die metra für sich gedruckt werden. Es sei hier ein für allemal bemerkt, dass die ags. metra durchans nicht unabhängig für sich bestehen. Ihre richtige stelle ist zwischen den prosaabschnitten der übertragung des Boetius. Nur aus äusseren gründen werden sie von den herausgebern separat gedruckt. Dass sie nicht für sich bestehen geht auch daraus hervor, dass nicht selten in den metren auf die vorhergehende prosa bezug genommen wird, und zwar nicht nur so, dass der betreffende ausdruck auf $\mathbf{B}$ beruht, sondern auch so, dass er eigens vom verfasser eingeschoben wird. Duch diese stellen werden weiter unten $\mathrm{zu}$ besprechen sein. 
anwendung des wortes, und diese findet sich im 25. metrum, welches beginnt:

'Zehêr nu ân spell be pôm ofermôdum',

wörtlich aus B entlehnt. Hier beruht der gebrauch von spell nur auf einer nachlässigkeit des verfassers, der unmittelbar vorher geschrieben hatte:

' $\partial \mathrm{a}$ se Wisdom $\gamma_{a} \gamma_{i s}$ spell areht hæide, $\gamma_{a}$ onzan he eft sinzan and Jus cwær'.

Man sieht daraus, dass Homer nicht der einzige dichter ist, der zu zeiten schläft. Doch kann diesem einen falle neben den 33 oben angefubrten keine grosse bedeutung zugeschrieben werden.

Wir konmen nun zu dem zweiten argumente, welches Wright gegen die verfasserschaft des königs vorbringt. Dasselbe ist aus dem geringen dichterisehen werte der metra entnommen:

'Had they been written by the king, they would certainly have possessed some of the higher characteristics of Anglo-Saxon poetry'.

Hier betreten wir einen boden, der in den augen mancher leute als besonders gefährlich gilt. In diesen tagen hochentwickelter philologischer kritik ist literarische würdigung bei vielen fast in miskredit gekommen. Charitius ', in seiner untersuchung uber den angelsächsischen Guthlac, lehnt es sogar ausdrucklich $a b$, sich auf irgend etwas derartiges einzulassén, da man bei der subjektivität derartiger kritik zu einem wirklichen resultate nicht kommen könne. Man könnte hier auch an die widerstreitenden urteile erinnern, die oft von verschiedenen kritikern tber ein und dasselbe gedicht gefällt worden sind. Hielt nicht Thorpe das werk, welchos unter dem namen Crist bekannt ist, für eine unzusammonhängende reihe unbedeutender religiöser gedichte, die viel zu gewöhnlich seien, als dass sie die mithe einer ernstlichen untersuchung verlohnten? Und hatte nicht Dietrich, den man mit recht den entdecker des Crist nennen könnte, eine ganz andere, unendlich höhere meinung davon? 'Thorpe ${ }^{2}$ widerum schäzte die von ihm veröffentlichte pariser tibertragung der psalmen sehr hoch: Dicendi generi

1 Charitius, Ueber die angelsächs. gedichte vom hl: Guthlac. S. diese zeitschr. II, s. 273.

${ }^{2}$ Libri psalmorum versio antiqua latina cum paraphrasi anglosaxonica etc. ed. B. Thorpc, Oxon. 1835, praef. s. V. 
maicstas inest gravitas et versus elegantia non vulgari concinnati sunt'. 'Ten Brink ${ }^{1}$ hingegen stellte die nämlichen psalmen vom dichterischen gesichtspunkte aus ziemlich tief. Ganz ähnlich in bezug auf unsere metra. Fox hält sie für ein ächtes muster angelsächsischer dichtkunst, und Tupper, ibr tibersetzer, gerät fast bei jedem stück in ekstase über vermeintliche dichterische schönheiten. Sein ganzer commentar fast ist ein strom bewundernder begeisterung, vor der jeder einwand des profanen kritikers als eine art literarischen hochverrats erscheint. Auf der anderen seite steht Wright's ansicht über die metra, die wir schon oben kenuren gelernt haben. Ten Brink bemerkt: 'Grosse poesie wird man nach dem gesagten in den metren nicht erwarten dürfen', und Swcet erklärt, dass sie fast jedweden dichterischen wertes baar seien.

Wie sehr aber auch derartige widersprechende urteile den verächtern literarischer kritik stütze zu verleihen seheinen, so sind sie doch keinesweg's hinreichend; die berechtigung derselben zu erschïttern. Dass naturen vorhanden sind, denen es an der hier nötigen füligigkeit gebricht, ist allerdings eine unleugbare tatsache. Es gibt eine physische farbenblindheit und es gibt eine ästhetische farbenblindheit. Es soll nicht gesagt werden, dass Thorpe daran gelitten hat. Aber, mit aller anerkennung seiner grossen verdienste um die angelsächs. literatur, wird man doch immerhin sagen können, dass der sinn für das schöne bei ihm nicht $z u$ besonders hoher entwicklung gelangt war. Aber auch noch andere umstände können sich der gerechten wïrdigung einer dichterischen produktion hindernd in den weg stellen. In dem besondern falle, angelsächs. poesie, kann mangel an vertrautheit mit dem ganzen umkreise ihrer schöpfungen ein ernster nachteil sein. Wer nicht durch ausgedehnte lektiure sich in die eigentiimliche form, in welcher dieses volk das schöne zur darstellung brachte, so zu sagen con amore versenkt hat, der kann sich über den wert eines einzelnen gedichtes sehr leicht täuschen. Und endlich ist die frage uber den poetischen wert der metra durch den umstand verdunkelt worden, dass es sich hier um könig Aelfred handelt. Die bewunderung die man diesem manne entgegen bringt, hat das urteil mehr als eines kritikers getrübt. Ich unterschreibe

${ }^{1}$ ten Brink, Geschichte der engl. Literatur s. 61. 
von herzen die meinung Freeman's 1 , der den könig widerholt als einen der vollendetsten charakteren in der geschichte bezeichnet. Ich bewundere seinen seelenadel, seine wahrheitsliebe, die fast einzige art, in welcher das leben des helden und die tätigkeit des gelehrten, des volksbildners bei ihm vereinigt erscheint. Doch ist nicht zu ersehen, was alles dies mit der ästhetischen würdigung seiner poesieen $\mathrm{zu}$ tun hat, wie sich diese verschiedenen elemente mit einander verwechseln lassen. Tatsache ist, dass das gefühl der bewunderung für den könig veranlassung gegeben hat sowol zu dem irrtume von Tupper wie zu dem von Wright. Nach der anschauung des ersteren, der in hohem grade an dem übel zu leiden scheint, welches Lord Macaulay irgendwo als 'lues Boswelliana' bezeichnet, muss könig Aclfred notwendiger weise ein grosser dichter sein, weil er in jeder andern beziehung gross ist. Nach Th. Wright linwiderum kann er unmöglich der verfasser der metra sein, weil sie, ein armseliges werk vom literarischen standpunkte aus, zu seiner anschauung vom könige als einem klassischen schriftsteller nicht stimmen. Beide anschauungen aber beruhen auf trugschliussen. Beide anschauungen vermengen elemente, die keinen zusammenhang mit einander haben. Das beispiel Ludwig's XIV., der, als sittlicher charakter allerdings nicht wert ist, Aelfred die schuhriemen zu lösen, mag zeigen, dass ein könig in mancher hinsicht gross sein und seinem eignen zeitalter sogar als ein idealer typus des königtums erscheinen kann, und dabei doch im stande ist, schlechte verse zu schreiben. Und das beispiel Cicero's ist ein schlagender beweis dafür, dass poesie und prosa zwei sebr verschiedene gebiete sind, dass man sehr wol auf dem einen glänzendes leisten und auf dem andern fiasco machen kann.

Doch lassen wir einstweilen die verbindung des namens Aelfred's mit den metren bei seite. Fragen wir uns: was sind sie, an und für sich betrachtet? Welchen dichterischen wert haben sio in den augen des unparteiischen kritikers? Die antwort auf diese frage kann nur die sein, dass sie fast alles dichterischen verdienstes baar sind, wie Sweet sich ausdrickt, und wenn Heinzel, in seiner schrift über den stil altgerman. dichtung, ihrer auch nicht einmal erwähnung tut, weil er sie

1 Freeman, Hist. of the Norman conquest, 0xford 1870, I, s. $48 \mathrm{ff}$. 
nicht als poesie im eigentlichen sinne des wortes anerkennt, so kann man ihn darum nicht tadeln. Man braucht nur $\mathrm{C}$ mit B zu vergleichen, um obiges gesammturteil gerechtfertigt zu finden. Schon die geradezu sklavische art, mit der sich der verfasser von $C$ an die worte und wendungen von $B$ hält, indem er nicht selten eine zeile ohne änderung herübernimmt ${ }^{1}$, in der regel aber sich begnïgt, lahme und bedeutungslose fullwörter einzusetzen; namentlich erweiternde ausdehnung des subjektes und objektes, solche zigge sind ein hinreichender beweis daftir, dass der flug der phantasic dem verfasser fremd war und dass er gänzlich ausser stande war, frei über den allitcrierenden rhythmus zu gebieten. Hier und da trifft er wol einmal eine verhältnissmässige glückliche wendung, eine erinnerung aus dem reichen schatze angelsächs. dichtkunst. Aber diese fälle sind 'rari nantes in gurgite vasto', welche die uiber das ganze sich lagernde dichterische mattheit nur um so deutlicher hervortreten lassen. Allerdings vermag ich nicht, mich der ansicht von ten Brink und Rieger anzuschliessen, nach denen schou der charakter der metra des Boetius eine poetische behandlung in angelsächs. sprache nicht zugelassen hätte. Wenn wir nicht das an dichterischen schönheiten so reiche angelsächs. gedicht Phönix besässen, so könnte jemand ebenso gut sagen, dass die eleganten disticha, die diesem gedichte zu grunde liegen, sich für angelsächs. poesie nicht eigneten. Alles konımt auf den eigentümlichen geist des dichters an. Vielleicht findet ten Brink's bemerkung auf das eine oder das andere der metra anwendung. Aber im allgemeinen ist ihr inhalt sebr mannigfacher art. Sie erstrecken sich auf gegenstände, die nicht selten, und zwar mit erfolg, von angelsächs. dichtern behandelt worden sind. Oder zeichnen sich diese nicht besonders in der darstellung des seelenschmerzes, des kummers aus? Finden sie nicht eine sehr glückliche und ergreifende sprache, wenn es sich darum handelt, Gottes macht und herrlichkeit zu preisen oder die gewaltigen dieser erde zu schildern? Ja, ich bin sehr geneigt anzunehmen, dass ein wahrer dichter, selbst in den fällen den rechten ton angeschlagen haben

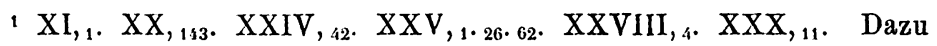
noch VI, ${ }_{8}$. XXI, ${ }_{10}$. XXIV, ${ }_{63}$, wo die änderung eine äusserst unbedeutende ist. 
würde, wo der inhalt sich gegen poetische behandlung zu sträuben scheint. Freilich würde dann der lateinische text nicht unbeträchtlich zu ändern gewesen sein. Der angelsächs. dichter musste sich begnïgen, die allgemeinen leitenden gedanken festzuhalten, und ihnen eine individuelle ausprägung geben. Etwas derartiges aber war für den verfasser von C unerreichbar. Selbst in den metren, deren inhalt erzählender art ist, und daher dic möglichkeit einer epischen behandlung darbot, selbst da ist der stil so matt und ungeschickt, wie irgendwo anders. Selbst dieser aufgabe war der umdichter nicht gewachsen.

Wie schwach aber auch die metra sein mögen, man darf nimmer zugeben, dass sie aus diesem grunde nicht von könig Aelfred stammen können. Nur dann würde dies annehmbar sein, und selbst da nur bedingungsweise, wenn könig Aelfred irgend ein gelungenes poetisches werk hinterlassen hätte. Dies ist aber nicht der fall. Ohne paradox zu sein, wird man sehr wol sagen können: Die metra sind von könig Aelfred, nicht obgleich, sondern gerade weil sie poetisch nicht geraten sind, gerade weil sie so sind, wie sie sind. Die metra tragen durchweg das gepräge eines lehrhaften geistes, wie er mit wahrhafter poesie sich nicht verträgt. Der verfasser scheint sich als eine art lehrer zu fühlen, er scheint sich immer daran zu erinnern, dass er zu einem ungebildeten volke spricht, bei dem er nicht eben viel voraussetzen darf. Daher macht er oft halt, um eine erklärung einzuflechten. Er bemuiht sich sichtlich, so verständlich als nur irgend denkbar zu reden. In diesem streben verdünt er seine gedanken oft in ganz unleidlicher weise, mit einer steifheit und ungelenkheit im stile, dass man sofort sieht, der verfasser ist im reiche der dichtkunst nicht zu hause.

Ein aufmerksamer leser der prosawerke Aelfred's kann nicht zweifeln, dass seine ganze begabung und beanlagung nicht nach der seite der kunst lag, die jemand, der sich darauf verstand, charakterisiert als 'of imagination all compact'. Sie verraten einen geist, der mit heissem bemühen nach. wahrheit ringt, der es sich zur aufgabe setzt, sie andern mitzuteilen, einen geist, dessen bestreben es ist, bildung zu erwerben und zu verbreiten, um dadurch sein volk zu heben, einen ehrlichen und nüchternen literarischen arbeiter. Seine mühsame tätigkeit 
als bearbeiter lateinischer prosatexte war nicht die sache eines phantasiemenschen. Sie hatte ibren ursprung in seinem leidenschaftlichen wahrheits- und wissensdrange. Die poesie konnte das gebiet einer solchen natur unmöglich sein. Und wenn man dazu noch die unruhen bedenkt, die seine regierung einleiteten und den .grössten teil derselben erfüllten, die allezeit bedrohte lage seines vaterlandes, seine ausgedehnte tätigkeit auf dem gebiete der verwaltung und gesetzgebung, und - last, not least jenes eigentümliche körpérliche leiden, welches er wie 'einen dorn im fleische' empfunden haben muss, so kann man sich nicht wundern, dass seine allgemeine geistige richtung mehr auf das utile, als auf das dulce gieng. Was war nun aber der gedanke des königs, wenn er als dichter vor sein volk trat? Ich gestehe, ich bin durchaus nicht geneigt, ibm deshalb den vorwurf der anmassung oder der unbescheidenheit zu machen. Es muss sich eine erklärung finden lassen, die mit dem charakter Aelfred's nicht im widerspruche steht. Und diese erklärung bietet sich zwanglos in der beschaffenheit des originaltextes selbst. Nachdem der könig es sich einmal vorgenommen hatte, seine landsleute mit demselben bekannt zu machen, musste er ihnen wol oder iibel die metra in poetischer form geben, so wenig neigung er auch selbst für diese aufgabe fuihlen mochte. Wenn anders er seinem lat. autor volle gerechtigkeit widerfahren lassen wollte, konnte er sie nicht umgehen. Und ubrigens hat er diese notwendigkeit von vornherein eingesehen. Dies geht deutlich genug aus den worten 'leod, $z y d$, fitte' hervor, mit denen er oft genug die entsprechenden prosaabschnitte bezeichnet. Dieselben würden ganz und gar nicht am platze sein, wenn sie sich auf wirkliche prosa bezögen. Sie lassen sich nur unter der voraussetzung erklären, dass der verfasser, von dem ersten augenblicke an, wo er seine übertragung unternahm, die feste absicht hatte, den metris diejenige form zu geben, die ihnen zukam. Dass or seiner aufgabe schliesslich nicht gewachsen war, kann die frage über die autorschaft durchaus nicht irgendwie beeinflussen.

Es eriabrigt noch die prüfung des dritten und letzten beweises von'Th. Wright. Derselbe gründet sich auf die armseligen klassischen kenntnisse des umdichters. Wright hat hier namentlich zwei schnitzer im auge, durch welche, wie er 
glaubt, der text arg verunstaltet worden ist. Met. XXVI, ${ }_{7}$ ist nämlich von Odysseus gesagt:

he wæs brâcia pîoda aldor

and Rêtie rîces hirde,

während die prosalubertragung liest (XXXVIII, 1$)$ :

' $\partial a$ dioda waron hatene Ioracize and Retie'

Die andere stelle findet sich metra $\mathrm{XXX},{ }_{3}$, wo Homer genannt wird:

Firziles frêond and lârêow,

während man im prosatexte liest:

'se wæs Firzilies lareow'.

Sind diese gründe wirklich genügend, um auf sie hin die metra Aelfred abzusprechen? Ich antworte: nein. Was das erste beispiel anbelangt, so ist natürlich von vornherein die möglichkeit nicht ausgeschlossen, dass Aelfred selbst' Iðacize schrieb, und das irgend ein puristisch verbessernder schreiber daraus Đracia machte. Indess selbst wenn das letztere von Aelfred herrührt; so liegt doch darin durchaus nichts bedenkliches. Gewiss würde die nachlässigkeit schwer in's gewicht fallen, wenn wir an Aelfred's zeitalter denselben maassstab anlegen köunten, wie an dieses unser neunzehntes jahrhundert. Dass Thracien und Ithaca nicht beliebig verwechselt werden können, durfte selbst einem quartaner nicht unbekannt sein. Es ist aber ein durchaus unwissenschaftlicher anachronismus, ein zeitalter mit dem maasse eines andern zu messen. Man mache sich nun einen-deutlichen begriff von dem allgemeinen zustande der bildung unter den Angelsachsen jener zeit, und man wird den fraglichen fehler im grunde sehr erklärlich und verzeiblich finden. Aehnliches kommt damals auch sonst vor. Ich erinnere nur daran, dass in der einleitung zur angelsächs. chronik gesagt wird, die Briten kämen aus Armenien, und zwar findet sich diese lesart in allen drei hs. (Cott. Tib. B. IV, Bodl. Laud. 636. Cott. Dom. A. VIII), wohingegen Bæda, aus dem das betreffende stück genommen ist, Armorica liest.

Uebrigens ist es nicht unmöglich, dass in unserem falle die vertauschung auf einer absicht beruht. Natürlich war dies nicht das bedürfuiss der alliteration. Denn als ein ausländisches mehrsilbiges wort konnte I-ðacige sehr wol mit ठioda alliterieren. $^{1}$ Aber vielleicht zog der verfasser T'bracien vor

1 Rieger, Alt- und angelsächs. Verskunst, Halle 1876, s. 11, anm. 
als einen geographischen namen, der damals verhältnissmässig besser bekannt war als Ithaca, ein wort, welches selbst für die gebildeten seiner landsleute nicht weniger fremdartig geklungen haben muss als z. b. 'Tapaling oder Amarakanthak für einen gebildeten Europäer des neunzehnten jahrhunderts. In dem bestreben, so verständlich zu schreiben, als die natur seines textes es überhaupt gestattete, mochte er Thracien für Ithaca unterschieben. Ein fall von schlagender ähnlichkeit findet sich in der prosaübertragung des Boetius 7, ${ }_{2}$. Da wird Crōsus als könig von Griechenland bezeichnet, während im lateinischen texte steht: regem Lydorum. Soll man etwa daraus schliessen, dass die prosaübertragung des Boetius nicht von Aelfred herrührt? Es sei hier nur noch dies bemerkt, dass für Aelfred's geographische begriffe Thracien allerdings ein griechisches land war, wie es ja in der tat damals zum byzantinischen reiche gehörte. So begreift er in dem einleitenden kapitel seines Orosius, nachdem er von Moesien, Thracien und Macedonien gesprochen hat, diese länder unter der allgemeinen bezeichnung: 'Jas land syndon Creca lande'. Und wir wissen positiv, dass er diese anschauung auch auf alte zeiten uberträgt; denn in der geschichte von Orpheus und Eurydice (Boetius 26,6 ) liest man die worte: 'Aracia, sio woes on Creca rice', und zwar sind diese letzteren worte ein erklärender zusatz des königs selbst. Es war im grunde doch gar nicht so absurd, wie Th. Wright anzunehmen scheint, einen griechischen ftirsten, Odysseus, mit einem griechischen lande in verbindung zu bringen. Ein argument gegen die authenticität der metra lässt sich daraus nicht herleiten.

Das nämliche gilt in bezug auf das andere symptom einer, wie Wright annimmt, groben unwissenheit des verfassers der stabreime: Homer, der freund des Vergil!

Hier kann man nun in der tat der ansicht sein, dass der verfasser das wort freond als eine passende alliteration zu Firzilies einfugte, um seinen vers auszufullen. Ist es aber ausgemacht, dass der text dadurch so gar sehr entstellt wurde? Ich gestehe, ich vermag dies nicht zu sehen. Denn mit demselben rechte, mit dem Aelfred den Homer als lehrer des Vergil bezeichnet, mit demselben rechte kann er ibn auch den freund desselben nennen. Es fragt sich nur, wie sind die beiden ausdrüicke $\% u$ verstehen. Versteht man den ersten in übertragenem 
sinne, nun, so ist dies natürlich auch dem zweiten gegenuber erlaubt. Die stelle würde dann bedeuten: Homer, dessen dichtung Vergil die entwickelung seiner eignen dichterischen fähigkeiten verdankt und mit dem er sich daher durch bande der liebe und zuneigung verbunden fuhlte. Vielleicht spielt ten Brink auf etwas derartiges an, wenn er bemerkt: 'Die misverständnisse der englischen poesie, die man der englischen prosa zur last legt, lösen sich bei genauerer prüfung in einer weise auf, die auf den dichter sogar ein günstigeres licht wirft als auf den prosaiker'. Aber freilich, ob es gestattet ist, die schwierigkeit so gar sehr günstig für den verfasser hinweg zu erklären, dass ist doch sehr fraglich. Nach dem eindrucke, den ich aus dem studium der Aelfred'schen schriften gewonnen habe, ist es kaum zulässig, die worte lareow und freond in einer derartig vergeistigten bedeutung aufzufassen. Das ist nicht die einfache und klare art, welche Aelfred's stil kennzeichnet. Nichts liegt ihm ferner als glänzende concetti, euphuistische wendungeu. Es durfte doch weit mehr dem standpunkte jener zeit entsprechen, wenn wir lareow sowol als freond in dem eigentlichen sinne verstehen, so dass dann einfach ein historischer irrtum vorläge, durch den die beiden dichter chronologisch zusammengebracht wären. Es ist ja nicht wol denkbar, dass die Angelsachsen jener zeit eine sehr klare vorstellung von Homer gehabt haben. Wir wissen zufällig, dass der angelsächsische glossator des Aldhelm'schen buches De. Virginitate ihn als eine art von sophisten oder redner ansah. ${ }^{1}$ Und der angelsächsische bearbeiter des Apuleianischen Herbariums nennt ihn einmal 'se moera ealdor'. ${ }^{2}$ Aelfred selbst fand allerdings den Homer im Orosius erwähnt $(1,7)$, doch gab ihm diese stelle keinerlei anhalt tuber die zeit, in welcher Homer lebte. Soweit ich zu sehen vermag, spricht durchaus nichts gegen die gegebene interpretation, und da sie auf freond ebenso wol wie auf lareon anwendung findet, so ist kein grund vorhanden, deswegen die autorschaft des königs zu bezweifeln.

1 Die ags. glossen im briisseler codex von Aldhelm's De Virginitale, ed. Bouterwek in Haupt's ztschr. f. d. a. 1853 s. 463: Homerum | wordsnoteran; vgl. s. 459 sophisma | wordsnoterung; s. 481 oratores (gl. rhetores, grammatici) wordsnotere.

2 Leechdoms, Wortcunning and Starcraft of early England, ed. by Cockayne, I, London 1864, s. 108. 
Doch hier möchte ein etwaiger irrtum abzuwebren sein. Wenn J. Grimm ${ }^{1}$ bei seiner vermutung, dass die form Elene für die bekanntschaft des angelsächsischen dichters mit einem griechischen texte der legende spricht, auch an den ausfall der anlautenden aspirata denkt, so würde die schreibung Omerus im metrum und im prosatexte, wie auch in der Orosiusbearbeitung, ebenfalls die bekanntschaft Aelfred's mit dem Griechischen bedingen. In der tat scheint diese ansicht hier und da gehegt zu werden. Ohne grossen nachdruck auf eine stelle bei Th. Wright ${ }^{2}$ zu legen, der einmal bemerkt:

'We might bring many passages together which seem almost to prove that Homer continued to be read in the schools till the end of the $13^{\text {th }}$ century',

ist hier besonders an Thorpe zu erinnern. Dieser scheint in der tat anzunehmen, dass Aelfred den Homer im griechischen urtexte las. Denn in seiner Orosiusausgabe bemerkt er gelegentlich der worte ' $\partial$ cet underiende folc Aethiopiam' $\left(1,{ }_{2}\right)$ :

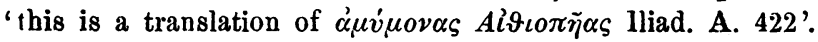

Natürlich kann Thorpe nur meinen, dass Aelfred hier eine klassische reminiscenz einfügte; denn der lateinische text enthält kein dem underiende entsprechendes wort, so dass dieses ein selbständiger zusatz Aelfreds ist. Derselbe findet aber eine ganz einfache erklärung, ohne dass man deswegen zu der annahme zu kommen braucht, Aelfied verstand Griechisch. Unbedeutend wie dieser zug scheinbar ist, so erkennt man doch daraus das wahrhaft menschenfreundliche herz des königs. Als er im Orosius las, dass die königin Semiramis die Aethiopier ungerechter und grausamer weise mit krieg uberzog, da empfand er eine regung des mitleids, und dieses gefubl fand seinen ausdruck in dem worte underiende. Dass Aelfred von der griechischen sprache nichts verstand, bedarf flur den kenner seines lebens und seiner schriften kaum einer darlegung. Wir brauchen nicht mit Gidel ${ }^{3}$ zu sagen: 'Alfred ne connaissait pas le grec, puisqu'(!)il a traduit du latin en saxon les fables d'Esope'. Wir würden ubel daran sein, wenn wir auf dieses argument

1 Andreas und Elene, herausg. von J. Grimm, Cassel 1840. Vorrede s. LII.

2 A. a. o. I, s. 40.

3 Nouvelles éludes sur la littérature grecque moderne, par M. Ch. Gidel, Paris 1878, s. 188. 
angewiesen wären. Die sache ist an sich so klar, dass eine widerlegung Thorpe's gänzlich überflüssig wäre.

Noch eine bemerkung in bezug auf die schreibweise Omerus und Elene. Es lässt sich nachweisen, dass es im Angelsächsischen eine weitverbreitete, fast ausnahmslos angewante regel war, dass die anlautende aspiration griechischer worte oder worte von angenommener griechischer herkunft wegfiel, wenn diese in der einheimischen rede gebraucht wurden. ${ }^{1}$ Dies lässt sich durch zahlreiche belege erhärten ${ }^{2}$, neben denen nur wenige ausnahmen zu verzeichnen sind. Die erklärung dieser eigentümlichkeit ist nicht

1 Fritsche, in dieser zeitschrift bd. II, s. 45i, bemerkt: 'Das $h$ der lat. namen wurde im Angelsächs. nicht ausgesprochen'. Auch für die lat. namen war die regel nicht ausnahmslos. So schreibt Orosius 6, ${ }_{37}$ Honorius.

2 Aelfred selbst schrieb Elena, obwol er Helena in seinen lat. texten fand: Oros: $1,{ }_{11} \cdot 6,_{4 \cdot 30}$. Bæda 1, ${ }_{8}$. Dasselbe wort findet man noch am anfang des 13. jahrhunderts so geschrieben. Es kommt nämlich ziemlich häufig im Lazamon vor, und zwar uiberwiegt in beiden handschriften die schreibung ohne $h$ entschieden. Normännischer einfluss fieng damals eben an, die heimische ïberlieferung zu durchkreuzen. Ferner schreibt Aelfred Ercol (oder Ercules, auch Erculus), wo seine lat. texte Hercules boten : Oros. $1,_{1}(2 \mathrm{mal}){ }_{10} .3,{ }_{9 \cdot 11}(2 \mathrm{mal})$, Boet. 16, ${ }_{2} \cdot 39,{ }_{4}$ (so auch noch bei Chaucer, Monk's 'I'ale 105). Elice für Helice: Oros. 3, 3 . Ellespon-

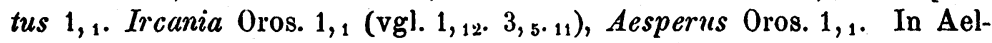
fred's Bæda (s. 493 ed. Wheloc) lesen wir: omeliorum. Dasselbe wort findet sich ohne $h$ in der überschrift der hs. Cott. Vespas. D. 14 (Wanl. Catal. s. 203), in einer angelsächs. glosse des 10. jahrhundert's (Wright, Volume of Vocab. I, Lond. 1857, s. 288) und im Ormulum (201 text). In Aelfred's Bæda findet man: 'ymen bec' = libri hymnorum (s. 494), und 'ervico metre' (ebenda). Unter den geschenken des bischofs Leofric (Wanl. Catal. s. 80) befanden sich ' $I I$ ymneras' und 'Liber Prudentii ymnorum'. In Liber Medicinalis (Wanl. Catal. s. 72) findet sich der name eeliotrophus, und eptafilon. Es gab im Angelsächs. einen 'Exameron' (Wanl. Catal. s. 72, 90), vgl. dazu das angelsächs. transscript eines griech. textes in der hs. Cott. Galb. A: 18, von dem ein teil abgedruckt ist im Lingard's Antiquities of the Anglo-Saxon Church, 1809. Mir nur zugänglich in einer deutschen übersetzung von F. H. Breslau 184i, s. 311). Noch bei Chaucer findet wan geschrieben: Omere (House of Fame 387), Ector (Troyl. 2, 171), armonye (Troyl. 5, 1826). Die constante schreibung Ĺrodes in der hs. des Heliand scheint eine angelsächs. hand zu verraten. (Sievers, in seinem Heliand, Halle 1878, einl. s. XV, spricht nicht davon, vgl. Schmeller, Heliand, proœmium s. IX). Ich weiss sehr wol, dass dies nicht die gewöhnliche schreibung des namens im Angelsächsischen ist. Die Evangelien, das Ev. Nic. nnd die meisten hss. der Sachsenchronik schreiben Herodes. Was hss. angelsächs. poesie anbelangt, so ist er 
schwer zu finden. Nachdem im jabre 664 Theodor und Adrian in England angekommen waren, wurde die griechische sprache etwas lebendiges für die gelelirten Englands und Bæda konnte in dem letzten teile seiner kirchengeschichte schreiben:

'Supersunt de eorum discipulis qui Latinam Graecamque linguam aeque ut propriam in qua nati sunt norunt'.

Und speziell vom bischof Tobias von Rochester berichtet er uns $\left(5,{ }_{23}\right)$ :

'Ita Graecam quoque cum Latina edidicit linguam ut tam notas ac familiares sibi eas quam nativitatis suae loquelam haberet'.

Solche verhältnisse blieben nicht ganz ohne einfluss auf die heimische sprache. ${ }^{1}$ Hier sei nur dies bemerkt, dass die in einen lebendigen besitz der griechischen sprache gelangten angelsächsischen theologen natürlich sehr bald bemerkten, wie wenig das einheimische $h$ dem griechischen spiritus asper lautlich entsprach, und da derselbe im Griechischen überhaupt nicht durch einen besonderen buchstaben ausgedrückt wurde, so zog man es bei der umschreibung in's Angelsächsische vor, ihn uberhaupt gar nicht besonders zu bezeichnen. Dies ist allem anscheine nach auf englischem boden eine allgemeine ortbographische regel geworden, die auch von solchen angenommen wurde, welche keine kenntnisse des Griechischen besassen. Daraus folgt, dass die auslassung des spiritus asper bei der angelsächs. transscription griech. worte für den betreffenden schriftsteller durchaus keine kenntniss des Griechischen bedingt.

Doch zuriuck zu unserem eigentlichen thema. Die zwei letzten einwürfe Wright's sind offenbar aus einer übertriebenen vorstellung von der altklassischen gelebrsamkeit des königs entstanden. Hier widerum hat die grösse seines charakters

meines wissens nur ein einziges mal Erodes geschrieben, nämlich Andr. 126, während man Jul. 293, Fat. Apost. 36 Herodes liest, was allerdings der alliteration wegen in Erodes zu ändern ist. Ueber dieses wort konnte man eben verschiedener meinung sein. Da es im neuen testamente vor$\mathrm{kam}$, so konnte es als griechisch angesehen und demgemäss behandelt werden, während andere es als nichtgriechisch betrachteten und daher mit $h$ schrieben. Jedenfalls weist die ausschliessliche schreibung ohne $h$ in der Cott. hs. des Heliand auf eine ags. schreibgewohnheit hin. Dieselbe war ja den ältern germanischen dialekten des kontinents unbekannt.

Anm. Auch noch in hss. des 15. jh. kommt die schreibung Elene vor, vgl. mein Altenglisches lesebuch II, s. 185 ff., z. 4. 26. 28. 32.38 .56 und sonst.

R. W.

$1 \mathrm{Vgl}$. Excurs. 
in der geschichte dazu verleitet, ihn mit vollkommenheiten auszustatten, die er unmöglich besitzen konnte. 1 .

Ganz naturgemäss musste Aelfred's kenntniss des griechischen und römischen altertums in vieler beziehung mangelhaft

1 Wright und Thorpe sind übrigens nicht die einzigen, die in dieser hinsicht zu weit gegangen sind. Von herausgebern, welche Aelfred die Alpen Muntgiop = mons Jovis nennen lassen (metr. Boet. 1, $8 \cdot 14$ ), kann man kaum sagen, dass sie sich den stand der klassischen bildung des königs wirklich vergegenwärtigt haben. Eigentümlicher weise hat sich diese so offenbar irrige ableitung seit dem Etymologicum Magnum bis auf unsere tage herab fortgepflanzt. Und doch spricht alles dagegen. Zuerst das allen germanischen sprachen gemeinsame gesetz, dass der bestimmende teil eines compositums stets an erster stelle steht. Zweitens die unmöglichkeit, dass lateinisches $v$ im Angelsächsischen zu $p$ wird. Das $v$ von Jovis wird im Ags. entweder $u$ (vgl. Bouterwek: Caedmon, pref. s. LII) oder $\mho$ (Aelfr. Boet. 35, $,_{4} .38,{ }_{1} .39,{ }_{4} ;$ Oros. $3,{ }_{9} ;$ metr. $26,{ }_{35} .47$ ) oder $f$ (Aelfr. Oros. 4, ${ }_{1.2}$; vgl. auch die form afitatores auf dem Frank'schen kästchen, bei Stephens, Old Runic Monum., London 1868, s. 475, und die form prafost $=$ propst bei Kemble, Cod. Dipl. VI, London 1848 , s. 154). Drittens die unmöglichkeit, dass Aelfred ein solches wort gebrauchte. Es liesse sich kaum denken, dass er es aus einem lateinischen antor genommen. Da aber kein lateinischer autor die Alpen jemals mons Jovis nennt, so müsste man diese bildung auf den könig selbst zuruickführen. Das ist aber unannehmbar. Ein so tief von christlicher anschauung durchdrungener mann wie Aelfred konnte nicht auf einen solchen ausdruck kommen. Denkbar würde er sein bei einem gelehrten der renaissance, von denen ja viele unbewusst eine antik-heidnische anschauungs- und darstellungsweise annahmen. Nicht so bei Aelfred. Uebrigens würde er sich damit der gefahr ausgèsetzt haben, von seinen landsleuten nicht verstanden zu werden, von denen doch nicht arzunehmen war, dass sie an Jupiter irgend welches interesse hatten. - Hier sei schliesslich noch auf eine verschiedene lesart bei Aelfi. Oros. 4, 2 aufmerksam gemacht. Da hat Bosworth: 'Jone wez zeworhte ofer (munt Jof) swa Jonne' und zwar sind die eingeklammerten worte aus der Laud. hs. genommen. Thorpe liest: 'Jonne wez zeworhte ofer munti. for swa. Jonne'. Die richtigkeit der lesart munt Jof $=$ mons Jovis ist sehr zweifelhaft. Unmittelbar vorher war gesagt: 'He com to Alpis Jam muntum'. Wie sollte für diese klare bezeichnung nun plötzlich gesetzt werden: munt Jof? Uebrigens kommt Aelpis $6 \mathrm{mal}$ in 1. kapitel des Orosius vor. Alles in allem genommen ist munt giop $=$ mons Jovis unhaltbar. Wie nun allerdings diese eigentümliche form $\mathrm{zu}$ erklären ist, ist mir selbst noch rätselhaft.

A n.m. Es scheint dem verf. entgangen zu sein, dass sich glossen finden (vgl. z. b. meine 'neuausg. der Wright'schen Glossarien s. 341. 355 u. s.): Alpium, muntzeofa; Alpes, munt iofes clifu. Das vorhandensein einer solchen erklärung des namen Alpes lässt sich also nicht hinwegbringen. An andrer stelle werde ich ausführlicheres darüber geben.

R. W. 
sein. Hat es denn für Wright nichts auffälliges, dass Aelfred in seiner unzweifelhaft ächten prosaübertragung des Boetius den Odysseus als könig von Raetien bezeichnet? Allerdings ist es ja nicht absolut sicher, dass Aelfred selbst ursprünglich für diesen irrtum verantwortlich zu machen ist. Denn die worte des Boetius vela neritii ducis müssen gewiss für manchen mittelalterlichen abschreiber eine unlösbare schwierigkeit enthalten haben und waren daher mancher verderbung ausgesetzt. Ja, in der alten berner handschrift der Consolatio ist die silbe ne von neritii unverkennbar zu dem vorhergehenden worte herubergezogen, so dass die stelle lautet: velani retii ducis. ${ }^{1}$ Es ist ja nicht unmöglich, dass Aelfred in der von ihm benutzten handschrift eine in ähnlicher weise verderbte stelle vorfand. Immerhin aber zeigt schon die blosse annahme dieser lesart, dass er nur eine dunkele kenntniss des klassischen altertums besass. Man sieht dies besonders deutlich, wie überhaupt seine mangelhafte kenntniss des Lateins, wenn man seine Orosiusiibertragung mit dem urtexte vergleicht, bei dessen bearbeitung er ja natïrlich besonders in gefahr war, versehen zu begehen. Ganz zu geschweigen davon, dass er den römischen eigennamen ziemlich übel mitspielt, nicht unälnlich Chaucer's Frankeleyne, der den M. Tullius Cicero in zwei oder drei verschiedene personen zerlegt. ${ }^{2}$ Die misverständnisse sind da ganz eigentiumlicher art. So liest man z. b. bei Orosius $5,{ }_{4}$ :

'(Fabius) fecit facinus etiam ultimis barbaris Scythiae, non dicam Romanae fidei et moderationi exsecrabile. Quingentis enim principibus eorum, quos societate invitatos deditionis jure susceperat, manus praecidit'.

Die scene ist in Spanien, zeit der viriathisehe krieg. Nachdem sich melırere feindliche stälte unterworfen hatten, machte Fabricius eine grosse zahl ihrer einwohner durch einen treubruch unschädlich. Dartiber die klage des Orosius. Wie ubersetzt nun Aelfred diese stelle? $\left(5,{ }_{2}\right)$ :

'se consul zedyde eallum Romanum $\partial a$ bysmerlicestan dæde $\partial a$ he aspeon of Sciððiun syxhund manna to him his zeঠoftena and $\partial_{a}$ hi

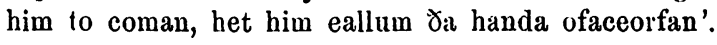

Man sieht, Aelfred fasst die rbetorische phraseologie seines textes wörtlich auf! Einfach und niichtern iv seiner denk- und

1 Peiper's Boetius, de Cons. Phil., Lips. 1871, s. 98.

2 Chaucer, Prolog zur erzählung des Frankel. 13-14. 
redeweise, hatte er kein verständniss fur rhetorischen pomp. Ein anderes eigentümliches misverständniss findet sich in seinem Oros. $5,{ }_{15}$. In dem lat. texte hatte Aelfred gefunden:

'Quinctilins Varus cum tribus legionibus a Germanis .... . funditus deletus est'.

Und doch übersetzt er:

'heora wear ær of ofslazen, buton tam consule anum'.

Wie erklärt sich dieser irrtum? Wol daraus, dass Aelfred nicht nur die obige stelle, sondern auch die im Orosius $\left(6,{ }_{21}\right)$ unmittelbar folgenđen worte unrichtig auffasste:

'quam reipublicae cladem Caesar Augustus adeo graviter tulit, ut saepe per vim doloris caput parieti collidens clamaret: Quinctili Vare, redde legiones'.

Aelfied scheint sich vorgestellt zu haben, dass Augustus diese worte an Varus selbst richtete, der bei ihm stand. Und er fährt dann fort:

'done consul he het ofslean'.

Das will uns kaum glaublich erscheinen, steht aber doch da. Auch im Boetius fehlt es nicht an ähnlichen irrtümern, im Boetius, den er, wie ich grund habe anzunehmen, bearbeitete, nachdem er schon jahre hindurch als ubbersetzer tätig gewesen war. ${ }^{1}$ So misversteht er in einem falle das adjectivum librum für einen eigennamen und bereichert dadurch die alte geschichte mit einer person namens Liberius. ${ }^{2}$ Manche seiner vertauschungen, das liesse sich unschwer zeigen, erklären sich nicht sowol aus dem streben, dem $\mathrm{zu}$ bearbeitenden texte eine art nationales gewand anzuziehen, wie man oft sagt, als aus einem einfachen misverständniss. Hierher scheint mir z. b. die beruhhmte ver-

- Ich kann mich hier anf eine begriindung dieser annahme nicht einlassen. Vielleicht finde ich später einmal gelegenheit, meine ansicht über die reihenfolge der werke Aelfred's zusammenhängend zu entwickeln.

2 Doch noch nicht so schlimm, als wenn Gotfrid von Viterbo, durch falsche lesung einer stelle des Paulus Diaconus, aus den worten hoc loco einen neuen geschichtsschreiber Toclacus schafft (Wattenbach, Deutschlands Geschichtsqu. i. M. II ${ }^{4}$, Berlin 1575, s. 227), oder wenn der verf. des provenzalischen Agnesspieles den sohn des präfekten Apodixes nennt, nach einem misverständniss seiner latein. quelle: 'Crudelissima omnium feminarum in filium meum voluisti apodixin tuae artis magicae demonstrare'. Uebrigens ist es selbst einem so bedeutenden kritiker wie Fabricius passiert, dass er aus der griechischen bezeichnung für rote tinte: $\mu \varepsilon \lambda \alpha^{\prime} \nu \iota \nu$ xóxxเvov, einen neuen griechischen autor: Melanios Coccinus herausgelesen hat. Vor wenigen jahren erst citierte ein französischer autor die werke 'd'un certain $M$. Verein de Stuttgart'. 
tauschung von Fabricius mit Weland zu gehöreu. Denn, wenn er das bewusste streben gehabt hätte, den inhalt des Boetius zu nationalisieren, so entsteht dic frage: Warum nahm er denn solche namen wie Papinianus, Parmenides, Euripides in seinen text nit herïber, mit denen seine landsleute doch kaum irgendwie bekannt gewesen sein können. Als einen irrtum möchte ich auch die tibersetzung von classica durch sciphere betrachten. ${ }^{1}$ Doch ich enthalte mich weiterer belege für eine tatsache, die jedem leser der Aelfred'schen schriften auffallen muss. ${ }^{2}$ Sein gedächtniss soll dadurch nicht verunglimpft werden. Haben wir doch bei keinem angelsächsischen schriftsteller eine so reiche gelegenheit, kenntniss antiker verhältnisse zu erforschen, wie gerade bei Aelfred. Wir sehen wol gelegentlich, dass Aelfric Cappadocien für eine stadt ansieht ${ }^{3}$, dass Cynewulf in einem seiner rätsel einen vogel namens Pernex durch ein misverständniss dieses lateinischen adjectivums fabriciert 4 , dass der ubersetzer der psalmen aus einem lateinischen futurum den ländernamen Metibor herauslas. ${ }^{5}$ Aber kein anderer angelsächsischer schriftsteller hat jemals den-ktihnen und grossen gedanken gehabt, werke wie Orosius und Boetius in der volkssprache zu bearbeiten, kein anderer daher hat sich so sehr der gefahr ausgesetzt, irrtiimer zu begehen. Doch der wert eines mannes bemisst sich nicht nach solchen lingen und am allerwenigsten eines mannes wie könig Aelfred. Seine verdienste sind zu gross, als dass sie dadurch geschmälert werden könnten. Wenn man nur einen augenblick an den zustand Englands zu jener zeit denkt, an den niedergang oder die vernichtung jener grossen brennpunkte der bildung, zu denen in den glücklichen

'Vgl. Aldh. Gloss. in Haupt's Ztschr. f. d. A. 1853, s. 467: classibus | bymum there.

${ }^{2}$ Mit unrecht findet Guizot auffällig (1. c. s. 168), dass Aelfred den Agamemnon als casere bezeichnet. Dies wort wurde in sehr weitem sinne gebraucht. So wird David in einem psalm casere genannt und derselbe name wird bei Lazamon auf Arthur angewant $\left(11,525,{ }_{13}\right)$. Kelle zu Otfrid 4, ${ }_{27 \cdot 10}$ citiert eine stelle aus Bæda: 'Jesus imperator credentium'.

3 Aelfric's Homilies ed. 'Thorpe 1844, s. 30.

4 Cynewulf's Ræts. 41, 66.

5 Libri psalm. versio etc. ed. Thorpe $1835,59,{ }_{5} .107,6$.

Anm. Aus den Glossarien hätten sich diese beispiele noch ausserordentlich vermehren lassen, glossen wie z. b. Dalmatie dære burze (also $=$ stadt) treffen wir zu dutzenden an.

R. W. 
tagen Theodor's und Breda's schaaren von wissensdurstigen schülern vom festlande aus gewallfahrtet waren, als zu den quellen der weisheit und gelehrsamkeit, so kann man sich nur wundern, dass Aelfred, ein laie, der erst im reifen mannesalter Lateinisch lernte, doch noch so viel erreicht hat. Die zeiten waren damals rorbei, wo, nach dem angelsächsischen sprichworte, eine frau mit einem kinde auf dem arm unversehrt von meer zu meer gehen konnte. Selbst zu friedenszeiten zwangen die ewig drohenden einfälle der nordischen piraten den könig, stets auf der hut zu sein. Eine weniger hochstrebende seele wurde dem übermenschlichen drucke erlegen sein. Mit einem anderen, weniger edlen geiste am ruder des staates wurde das schwach glimmende licht der kultur gänzlich erloschen sein. Aelfred's unvergänglicher ruhm ist es, das heilige feuer genäbrt und durch seine mühevolle literarische tätigkeit den arbeiten künftiger generationen vorangeleuchtet zu haben. Wir wissen, leider nur durch spärliche zeugnisse, dass bald nach Aelfred eine ausgedehnte prosaliteratur in der heimischen sprache aufkam. Diese literatur verdankt ihren ursprung dem könig Aelfred. Aelfric selbst fühlte sich gewiss bedeutsam angeregt durch das beispiel seines erlauchten vorläufers. So war die tätigkeit des königs reich an fruchtbaren keimen, und sein name lebte fort bis auf späte geschlechter als ein symbol für alles gute und edle und weise und grosse. ${ }^{1}$

Nachdem wir so Wright's ansicht ausfuhrlich widerlegt haben, bleibt noch die frage übrig, ob sich die authenticität der metra durch positive gründe stutzen lässt. In der tat fehlt es nicht an solchen. Hierher gehört vor allem die in prosa abgefasste vorrede, welche dem ganzen werke vorausgeht, und welche sich in beiden handschriften findet. Darin heisst es:

' $\gamma_{\mathrm{a}}$ he Jas boc hæfde zleornode and of Lædene to Enzliscum spelle zewende and zewohrte hi eft to leoðe swa swa heo nu zedon is'.

1 Vgl. Wülcker über die sprichwörter könig Aelfred's im 1. bande von Paul und Braune's Beiträgen. Dazu eine stelle einer ags. predigt in Wheloc's ausgabe von Aelfred's Bæda s. 169: 'Cyninzo zerist rihtwisnyss and wisdom; him is nama zesett of sodum reccendome gcet he hine sylfne and sioran his leoda mid wisdome wissize and wel zerihtloce; got folc bið zesceliz Jurh snoterne cyninz, sizefoest and zesundfull Jurh zesceadwisne reccend'. $\mathrm{Ob}$ der verfasser dieser zeilen bierbei nicht an könig Aelfred gedacht hat? 
Warum ten Brink diese vorrede als nichtaelfredisch ansieht, ist mir unersichtlich. Sie findet sich in beiden handschriften. Und sie trägt so offenbar den stempel der ächtheit, nicht nur in der phraseologie, die hier und $\mathrm{da}$ an eine andere vorrede des königs erinnert, sondern auch in ihrem ganzen tone. Die wahrhaft kindliche einfalt des gemuits, mit der sie geschrieben ist, die tiefe frömmigkeit, die sich darin ausdrückt, die liebenswürdige bescheidenheit, die gegen das ende hin hervortritt, das sind ächt Aelfredische züge. Ganz begreiflicher weise schickte er gerade sciner bearbeitung des Boetius eine vorrede voraus. War dies doch sein reifstes und in vieler beziehung auch sein wichtigstes werk. Machte es doch auf seine landsleute einen grossen eindruck. Es war so zu sagen das geistige vermächtniss, welches er seinem volke hinterliēss. Einem solchen werke, welches so voll von seinem eigenen geiste war, und auf welches er so viel sorgfalt verwant hatte, schickte er naturgemäss eine vorrede voraus.

Vielleicht könnte man gegen die authenticität der vorrede gerade den umstand geltend machen, dass darin die alliterierende ubertragung der metra erwähnt wird. Doch lässt sich dieser beweisgrund bei ernstlicher prüfung nicht halten. Nur dies geht daraus hervor, dass der könig selbst seine aufgabe dann erst als wirklich vollendet ansah, nachdem er die metra in poetische form umgegossen hatte. Erst dann setzte er dem werke die prosavorrede voraus. Vielleicht haben wir uns die sache folgendermassen zu denken: Nachdem er die prosatibertragung des Boetius beendet und seinen freunden gezeigt oder auch teilweise vorgelesen hatte, drangen diese in ihn, sie abschriften des werkes nehmen zu lassen. Der könig mochte ihren bitten nachgeben, wenn auch vielleicht widerstrebend, da er eben seine arbeit noch nicht als beendet ansehen konnte. Hierauf mag ein gewisser zeitraum verflossen sein, bis er muse fand, sie wider aufzunehmen. Inzwischen war die prosaübertragung bekannt und beliebt geworden. Ihr erfolg ermutigte natillich den verfasser, die worte der ersten übertragung so weit als möglich beizubehalten, als er daran gieng, die metra in versform zu bringen. Dieser endgiltigen form des werkes wurde dann die rorrede hinzugefligt. Solche natürlich, welche die erste ausgabe, wenn man so sagen darf, vorzogen, setzten ihr die vorrede der zweiten ausgabe vor. Bei einer solchen an- 
nahme fällt das obige bedenken gegen die vorrede selbstverständlich weg.

Wenden wir uns nun, nach der vorrede, zu den metren selbst. In der tat lässt sich ihre authenticität aus ihnen selbst erhärten. Zahlreich sind die hier anzuführenden beweise allerdings nicht, aber das ist ja nur natürlich, wenn man bedenkt, dass $C$ sich ausserordentlich eng an $B$ anschliesst. Zahlreich würden sie nur dann sein können, wcnn der zu grunde liegende text frei bebandelt worden wäre.. Dann würde es leicht sein, die unguis leonis, die spuren von Aelfred's originalem geiste, zu erkennen. Aber die metra enthalten äusserst wenig selbständiges, kaum einen gedanken, der nicht seinen entsprechenden ausdruck in $B$ bätte. Offenbar hat man sich durch diese wahrnehmung abhalten lassen, eine genaue vergleichung von $\mathrm{B}$ und $\mathrm{C}$ vorzunehmen. Natürlich können hier nur selbständige zusätze in betracht gezogen werden. Und doch lässt sich, aus diesen quantitativ allerdings sehr unbedeutenden zusätzen, nachweisen, dass der verfasser von $B$ identisch ist mit dem verfasser von C. Hierher gehört zuerst die stelle metr. 9, ${ }_{61}$ :

'pæ̂r wæs swî̀e sweotol, pæt we ş̂̉on oft, pæt se anwald ne dêð âwiht zôdes, zif se wel nele $\partial \mathrm{e}$ his zeweald hafay'.

Dieser gedanke gibt den schlusssatz von $\mathrm{B} 16,{ }_{4}$ wider, wo es heisst:

'Hu ne wæs zenoz sweotol $\gamma_{\mathfrak{~}}$ se anweald his azenes næs da se zod næs te he to com?'

Aber der dichter fugte hinzu: 'doet we saedon oft'. Wo sagte er es oft? Keine ähnliche stelle findet sich in dem metrischen teile, wol aber kommt derselbe oder ein ahnlicher gedanke mehrere male in dem langen prosaabschnitte, der unmittelbar vor dem bezüglichen metrum steht, vor. Da heisst es $16, \S 1$ :

'se anweald næfre ne bið zod, buton se zod sie ðe hine hæbbe'.

Und ebcnda §2:

'Hwat wenst $\gamma \mathrm{u}$ yonne hwæt zodes se anweald sie Jonne he on nane

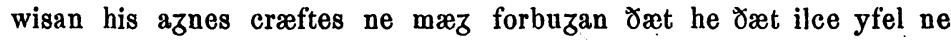

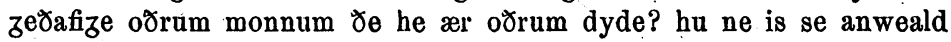
Jonne Jær nauht?'

Und ebenda § 3 :

'Hwæt hwenst $\gamma u$ ? zif se weor $\partial$ scipe and se anweald aznes zod wære and his selfes anweald hæfde, hwæðer he wolde Jam forcuðestum mannum folzian swa he nu hwilum deð?' 
Ebenda:

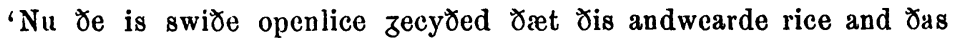
woruld zesælða and ${ }$ es anweald of heora aznum zecynde and heora aznes zewealdes nauht zodes ne sient'.

Ebenda:

' 3 if 'se anweald Jonne of his azenre zecynde and of his azenes zewealdes zod wære, ne underfenze he næfre da yfelan ac ða zodan'.

Ebenda:

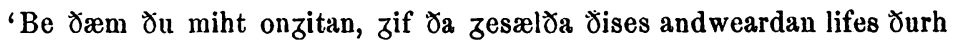
hie selfe heora selfie zeweald ahton and of heora aznum zecynde zode waron, Jonne woldon hi simle on Jam clifian ye him zod mid worhte'. Ebenda:

'Hit is nu hræðost to seczanne be eallum ðæm woruldzesælðnm $\gamma_{e}$

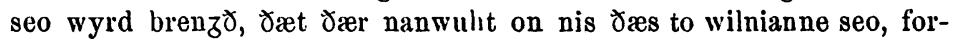

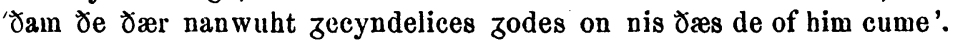
Das sind die betreffenden stellen, in ganzen sieben. Was sich daraus ergibt, liegt auf der hand. Indem der verfasser von $\mathrm{C}$ am ende des folgenden metrums zu dem nämlichen gedanken hinzufügt: ' Jeet we scedon of $l$ ', identificiert er sich offenbar mit dem verfasser des vorausgehenden prosaabschnittes, $d$. h. er ist könig Aelfred.

Ein ähnlicher zusatz findet sich am anfange des einundzwanzigsten metrums:

'Wel lâ! monna bearn zeond middanzeard

frîora âzhwilc fundie tô

pæm êcum zôde pe we ymb sprecað

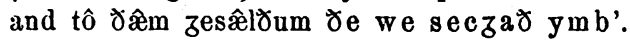

Hier ebenfalls identificiert sich der dichter des metrums mit dem verfasser der vorausgehenden prosa, d. h. er ist könig Aelfred.

Man wird nicht einwenden wollen, dass die fraglichen zusätze von dem unbekannten umdichter des 10. jahrbunderts absichtlich eingefïgt worden seien, um den glauben zu erwecken, dass seine arbeit das werk des königs sei. Dieser einwand wilrde psychologisch ganz widersinnig sein. Nur so riel sei hier bemerkt, dass, wenn ein fälscher wirklich die absicht gehabt hätte, seine verse als die Aelfred's auszugeben, er sie wol etwas häufiger und etwas in die augen fallender ausgefuhrt haben wtirde. Es muss daher die obige erklärung der beiden stellen, nach welcher der gebrauch der ersten person in einer bezugnahme auf die vorhergehende prosa in direkter und schlagender weise auf könig Aelfred als verfasser hinweist, als die einzige mögliche und natürliche angesehen werden. 
Neben diesem schlagenden beweise sei noch auf etwas andcres hingewiesen, auf eine eigentimlichkeit der darstellung Aelfred's, die auch an einer stelle der metra hervortritt. Ich meine den zusatz im 20. metrum, v. 169-175. Indem da der verfasser das verhältniss zu erklären sucht, in welchem die erde zu dem sie umgebenden wasser und zu dem den ganzen middanzeard einschliessenden himmel steht, gebraucht er das gleichniss von dem dotter inmitten des eies:
'pæin anlîcost pe on æze bið
zioleca on middan, zlîdeð $h w æ \gamma r e$
æz ymbûtan: swa stent eall weoruld
stille on tille, strêamas ymbûtan
lażuflôda zelâc lyfte and tunżla
and sîo scîre scell scrî̉è ymbûtan
dôzora zehwylce, dyde lanze swâ'.

Wenn man nun die prosaübertragung des Boetius mit dem lateinischen originale vergleicht, so sieht man, dass der könig diese art von, wenn ich so sagen darf, hausbackenen vergleichen sehr liebt. Charakteristisch für seine stellung als lehrer seines volkes, verschmäht er nichts, wenn es dazu dient, seinen gedanken klar und verständlich zu machen. Folgendes sind dio hierhergehörigen fälle:

16,,$_{1}$ 'Hu ne wile he Jonne don swa hy dydon and zit doð ealle

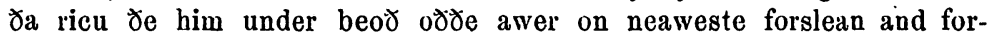

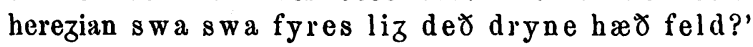

$20,,_{1}$ : wo er von dem unglücke spricht, durch ivelches der mensch zu dem wahren gute gebracht wird, fuigt er hin\%u: 'swa swa mid anzle fisc zefanzen bio'.

24, ${ }_{1}$ : Um zu erklären, dass alles glück von dem wahren gute abhängt, setzt er hinzu: 'swa swa ealle wæteru cumad' of $\gamma æ r e s x$ and eft ealle cumað to $\partial æ r e s æ '$.

$35,,_{3}$ fügt er am schlusse einer längeren abstrakten erörterung in ziemlich origineller weise hinzu:

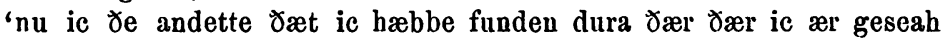
ane lytle cynan, swa ðæt ic uneaðe mihte zeseon swiðe lytellne sciman

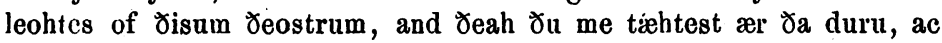
ic hire ne mihte mare aredian, buton dæt ic hire zrapode ymbuton jæt $\partial \mathrm{e}$ ic $\partial æ t$ lytle leoht zeseah twinclian'.

$36,,_{1}$ : 'se wisdom and eac oöre cræftas næbbað nan lof ne nænne weorठscipe on $\partial_{i s e}$ worulde, ac liczað forsewene s wa swa meox under feltune!'

$36,{ }_{7}:$ 'swa swa ælces huses wah bið fæst æzðer ze on ðære flore ze on $\gamma æ m$ hrofe, swa bið ælc zod on 3ode fæst, for $\gamma_{2 m}$ he is ælces zodes æzðer ze hrof ze flor'. 
$37,{ }_{1}$ vergleicht er ungerechte könige mit tollen hunden:

'se je hiora (scil. ذezna) welt, ne murnò nauðer ne friend ne fiend de ma de wedende hund'.

$37,{ }_{4}$ erweitert Aelfred in charakteristischer weise einen satz des Boetius. Dieser sagt von jemandem, der sklave seiner fleischlichen lüste ist: 'sordidæ suis voluptate detinetur'. Aelfred seinerseits begnügt sich nicht mit der übersetzung: 'he bio anlicost fettum swinum', sondern er fügt zu weiterer erklärung hinzu:

'خe simle willnað liczan on fulum solum and hi nyllað aspylizan on hluttrum wæterum, ac Jeah hi seldum hwonne beswemde weorठon, donne slea $\partial$ he eft on $\partial \mathrm{a}$ solu and bewealiviaj $\partial æ r$ on'.'

$38,{ }_{2}$ spricht er von dem plötzlichen zusammenbruch der macht der gottlosen und fiigt hinzu: "swa swa zreat beam wyrco hludne dynt ononne men læst wenad'.

$38,{ }_{4}$ wo gesagt wird, dass ein teil der gottlosen ewige strafen zu leiden hat, während der andere im himmlischen feuer gereinigt wird, fügt er hinzu: 'swa her biò sylfor'.

$38,{ }_{5}$ verweilt er ausführlich bei der ausmalung eines gleichnisses, welches sich im Lateinischen nur andentungsweise findet:

' $\partial u$ wæst $y_{\text {ret }} \partial_{a}$ men de habbað unhale eazan, ne mazan ful eaðe locian onzean $\partial \mathrm{a}$ sunnan jonne hio beorhtost scind, ne furòm on fyre ne on nanwuht beorhtes hi ne lyst locian, zif se æppel lef bið’.

$39,{ }_{1}$ vergleicht Aelfred die aufregung der seele durch hass mit der aufregung des meeres durch sturm:

'forhwi drefe ze eowru mod mid unrihtre fiounze swa swa yơ for winde $\partial a$ sæ hrerad'.

39, 4 : 'ne cymঠ' he næfre to openum ende buton he hæbbe swa scearp andzet swa Jæt fyr'.

Das allegorische gleichniss vom rade, welches Aelfred 39,7 einfüigt, un in drastischer weise die beziehung der geschöpfe zum schöpfer zu erläutern, ist zu ausgedehnt, um hier citiert zu werden. Mit seinem ausfiilhrlichen verweilen bei kleinen einzelheiten erinnert es an die lange allegorie Otfrid's iiber den rock Christi $\left(4,{ }_{29}\right)$. Es ist nicht ganz richtig, mit Fox zu sagen, dass 'this whole section is king Alfred's original production'. Derselbe wurde vielmehr dazu durch eine, allerdings nur kurze, stelle seines lateinischen textes veranlasst. Doch hat er es sehr ausgedehnt, vielleicht allzu sehr, und zugleich in eine populäre form gekleidet. Während bei Boetius das gleichniss einen streng wissenschaftlichen charakter hat, setzt Aelfred an die stelle der geometrischen figur des originals ein rad, um seinen gedanken dadurch anschaulicher zu machen.

1 Diese tiere scheinen bei den Angelsachsen keine unwichtige rolle gespielt $\mathrm{zu}$ haben. Vgl. H. Leo, Rectitudines singularum personarum, nebst einer einleitenden abhandlung uiber landansiedlung (und angelsächs. ortsnamen!), landbau, gutsherrliche und bäuerliche verhältnisse der Angelsachsen, Halle 1832, s. 128-129; vgl. Gesetze könig Ina's 44: 'zif mon aceorfe ænne treowe $\partial æ t$ mæze jrittizswina under standan and wyrò undyrue, zesylle sixtiz scillinza'. 
39, 11 vergleicht Aelfred den im besitz der gottlosen befindlichen reichtum mit einer art von arznei, die der arzt Gott ihnen eingibt, um dadurch ihre seelen zu heilen:

'se zoda læca, ðæt is 3od lacnaठ hiora mod mid ðam welan, wile jæt hi onziten hwonan him se wela come'.

41, 3 gebraucht der verfasser das gleichniss vom piloten, um den charakter der vorsehung zu erklären:

'swa swa zod scipstyra onzit micelne windhreose ær ær hit weorठe and hæt fealdan $\partial æ t$ sezl and eac hwilum leċzan yone mæst and lætan ya betinze, zif he ær $\partial w e o r e s$ windes bætte, wærnað he hine wið dæt weder'.

Aus diesen zallreichen beispielen dürfte wol zur genüge hervorgehen, dass in der tat die der sphäre des täglichen lebens entlehnten vergleiche ein charakteristischer zug von Aelfred's stile sind. Der vergleich mit dem eidotter im 20. metrun verdient daher beachtet zu werden. Wenn er auch selbstverständlich keine zwingende beweiskraft hat, so mag er doch immerhin dazu dienen, das auf anderm wege gewonnene resultat zu bekräftigen.

Schliesslich wäre noch zu fragen, ob vielleicht der sprachliche charakter der metra auf könig Aelfred als verfasser hinweist. Hier allerdings gestehe ich, vermag ich nur einige kleine beiträge zu liefern. Da die alliterierende übertragung sich in worten und wendungen eng an den prosaentwurf anschliesst, so können natürlich die ergebnisse nicht eben bedeutender art sein. Denn nur solche worte können hier in betracht kommen, die in der poetischen übertragung hinzugefugt sind. Und sodann. ist der angelsächsische philolog nicht in der glticklichen lage, ein Corpus totius Anglo-Saxonicitatis zu besitzen. ${ }^{1}$ Bos-

1 Bis dahin sind behauptungen ïber das vorkommen ags. worte mit vorsicht anfzunehmen. So sagt z. b. Sweet, in der vorrede zu seiner ausgabe des Hirdeboc: 'leorningcniht, for which Alfred employs jezn, has a distinctly late character'. Sweet ïbersieht, dass im Hirdeboc 25, 8 leorningcnihtas steht! Ferner sagt Sweet in seiner anzeige von Skeat's ausgabe der ags. und north. ïbertragung des Johannisevangeliums (Akad. 1879, Jan. 18th): 'the word hana had given way to cocc as early as the time of Alfred'. Doch hana findet sich Marc. 14, 30.68.72 (2 mal); Luc. 22, 34.60.61. Das compos. han-cred steht Aelfr. Bæda 4, 23 ; Natth. 14, 25 ; Marc. 14, 35. Aelfric's astron. Tractat (ed. Wright in den Popular Treatises on Science etc. s. 6, 14).

An m. Auch glossen sprechen gegen diese behauptung, vgl. z. b ein glossar aus dem 10. oder 11.jh. (in meiner neuansgabe s. 260) gallus = hana, während allerdings Aelfric's glussar gallus mit coc iibersetzt. R. W. 
worth's wörterbuch ist für philologische zwecke wenig verwertbar. Ja, wir besitzen noch nicht einmal ein Aelfred'sches glossar. So, wie die verhältnisse jetzt noch liegen, nuss fur die prosa wenigstens jeder sein eigener lexicograph sein.

Das wenige, was ich in bezug auf unsere frage beizubringen vermag, ist folgendes:

Das wort magister kommt zweimal in den metren vor: 13, 20 und $30,{ }_{4}^{4}$. Während es in der ersten stelle aus B entlehnt ist, ist es an der zweiten selbststïndig eingefügt. In der angelsächsischen poesie findet sich dies wort sonst nirgends. Dagegen ist es in Aelfred's schriften so häufig, und zugleich so ausserordentlich selten in der iibrigen prosaischen literatur, dass man es mit recht als ein Aelfred'sches wort ansehen kann: Bæda $1,{ }_{7 \cdot 27} \cdot 2,{ }_{20} .3,_{23} .4,_{2 \cdot 5 \cdot 27 .} 5,_{10 \cdot 20}$; Hird. Boc $109,_{14} \cdot 117,,_{6} .455,20$; Oros. $3,{ }_{9}$; Boet. $25.29,{ }_{2} .31,{ }_{1}{ }^{1}$ Dies wird um so auffälliger, wenn man beachtet, dass die angelsächsischen übersetzer der evangelien², welche das wort ziemlich häufig in ihren lateinischen texten ${ }^{3}$ funden, es offenbar vermiéden. Sie nehınen es auch nicht ein einziges mal heruiber, sondern geben es ausschliesslich durch lareow. Folyendes sind diese stellen: Matth. $9,_{11} .10,{ }_{24 \cdot 25} \cdot 12,{ }_{38} \cdot 17,{ }_{25} .19,{ }_{16} \cdot 22,{ }_{16} \cdot 24 \cdot 36 \cdot 23,8 \cdot 10.26,{ }_{18} ;$ Marc. $2,{ }_{16}$. $5,{ }_{35} \cdot 9,{ }_{17} \cdot 38 \cdot 10,{ }_{17} \cdot 20 \cdot 35 \cdot 12,14 \cdot 19 \cdot 32 \cdot 13,1 \cdot 14,{ }_{14} ;$ Luc. $3,12 \cdot 6,{ }_{40}(2 \mathrm{mal}) .7,_{41}$. $9,_{38} \cdot 10,{ }_{25} \cdot 11,{ }_{46} \cdot 12,{ }_{13} \cdot 18,18 \cdot 19,{ }_{40} \cdot 20,{ }_{21} \cdot 28 \cdot 39 \cdot 22,{ }_{11} ;$ Joh. $1,{ }_{38} \cdot 3,{ }_{2 \cdot 10} \cdot 8,{ }_{4}$. 11, 28. 13. 13.14. 20, 16. Ausserhalb Aelfred ist mir das wort nur ein mal aufgestossen, Aelfric Exod. 1, 11 , wo es dem lat. magister entspricht. In seinen homilien gebraucht Aelfric nur lareow. Diese tatsachen scheinen sehr dafür zu sprechen, dass magister kein eigentlich populäres wort im Angelsächsischen war, ungleich dem altdeutschen meistar, sondern lediglich auf gelehrter einführung durch könig Aelfred beruht. Das vorkommen in den metren deutet stark auf könig Aelfred als verfasser hin.

Die verbindung von hwoet mit hwuzu kommt nur zwei mal in den metren vor: 11, ${ }_{52}$ (welche stelle in Grein's Sprachschatz tibergegangen ist) und $20,{ }_{3}$. Während es in letzterem falle die in B gebrauchte diminutivform hwcethweguninga ersetzt, ist es in dem ersten falle ein selbständiger zusatz des umdichters. Dieser ausdruck hwothwugu (var.: -hwigu, -hwegu, -hugu) ist in Aelfred's werken ausserordentlich häufig:

1 Anm. Vollständig ist dieses verzeichniss nicht, so ist $\mathrm{z}$. b. die 'Epistola Alexandri ad Aristotelem' iiber'sehen. Vgl. diese zeitschr. bd. IV, s. $139 \mathrm{ff}$. (z. b. z. $3,7,58)$. R. W.
R.

2 Ohne zweifel ist die angelsächs. iibertragung der evangelien das werk mehrer männer. Hier sei nur darauf hingewiesen, dass Matth. $1-14$ incl. das wort phariseus stets durch sunderhalig gegeben wird, während $16-23$ das lat. wort ebenso constant beibehalten ist. Erst kap. 27 taucht sunderhalig wider auf. Vgl. Trench, $O \mathrm{n}$ the Author. Version of the New Test. London 1858, s. 48. Eadie, Hist. of the Engl. Bible, London 1876, II, s. 135,365 .

3 Als selbstverständlich nehme ich an, dass die angelsächs. evangelien auf dem lat. texte beruhen. 'Thorpe vergleicht allerdings gelegentlich das Griechische. 
Bæd. 1, 7.27 (2 mal) 2, 5.6. $3,_{8}(2 \mathrm{mal})_{9 \cdot 10} .4,_{3} .5,_{2}$; Hird. Boc. $71,_{22} \cdot 147_{1}$.

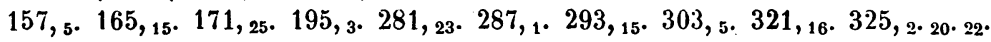

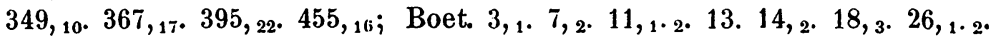
$32,{ }_{1} \cdot 33,{ }_{1} .34,,_{1 \cdot 4 \cdot 6}(2 \mathrm{mal})_{7}(2 \mathrm{mal})_{9 \cdot 10} \cdot 35,{ }_{1} \cdot 5 \cdot 26,,_{1 \cdot 3} \cdot 38,,_{3}(4 \mathrm{mal}) .39,{ }_{4}$ (2 mal) ${ }_{7}$, d. h. in ganzen $59 \mathrm{mal}$ in Aelfred's prosaschriften. (Wie mir herr prof. Wülcker sagt, ist es auch in den Soliloquien häufig.) Ebenso verbindet Aelfred gern hwuz $u$ mit hwylc: Bæda $2,{ }_{1}{ }^{1} 3,{ }_{2 \cdot 22} \cdot 4,{ }_{9 \cdot 22 \cdot 25 \cdot 27}$. $5,{ }_{2} \cdot{ }_{11} \cdot{ }_{14} \cdot 20$; Hird. Boc. $397,{ }_{26} \cdot{ }_{29}$, und mit $h u$ : Bæda $3,{ }_{27} \cdot 4,_{19 \cdot 27} \cdot 5,{ }_{4}$ (2 mal) 6 , und mit oelc: Oros. 3,,$_{7}$. Allerdings kommt nun das wort auch sonst vor, nämlich, was prosa anlangt, im Conf. Ecgb., öfters in den Leechdoms und in den Blickling Homilien, in gebundener rede aber nur zwei mal, in den metrischen psalmen $89,{ }_{15} .93,8$. Mit zwingender notwendigkeit fiihrt daher das vorkommen des ausdrucks nicht anf könig Aelfred. Doch mag er immerhin als zu den stileigentümlichkeiten desselben gehörig betrachtet werden.

Ealne, oder ealniz ist ein häufiges füllwort in den metren: $7,_{40.53}$. $10,{ }_{21} \cdot 21,{ }_{15} .22,{ }_{15} .28,,_{57} \cdot 69$. In der poesie findet es sich sonst nirgends. Was Aelfred's schriften anlangt, so habe ich es mir aus dem Hirdeboc zwölf mal notiert: $9{ }_{5} .239,{ }_{6} .263,_{16} .283,{ }_{5} .285,{ }_{17} \cdot 293,{ }_{14} \cdot 329,{ }_{17} \cdot 395,{ }_{29}$. $413,20 \cdot 29.421,{ }_{18} .453,9$.

Das wort ymbhoga findet sich zwei mal in den metren, während es an den betreffenden stellen von $B$ nicht steht: $7,{ }_{36} .53$. Es ist häufig im

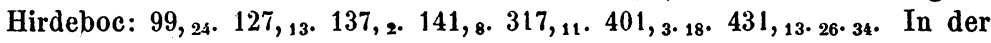
poesie kommt das wort ausser den metren nirgends vor.

Die verbindung morðum gefroege, metr. $20,,_{2}$ findet sich in angel sächsischen poesie nur noch in dem prolog Aelfred's zum Hirdeboc. Doch mag dies vielleicht mit lediglich äusseren gründen zusammenhängen.

Hiermit ist unsere untersuchung beendet. Fassen wir alles zusammen, so ergibt sich als endresultat folgendes:

Die argumente Wright's gegen die ächtheit der metra haben sich als pseudoargumente herausgestellt, d. h. die metra enthalten schlechterdings nichts, was dagegen angeführt werden könnte. Im gegenteil, dieselben haben uns elemente an die hand gegeben, durch welche es möglich wụrde, ibre ächtheit wol uber jeden zweifel sicher zu stellen. König Aelfred und niemand anders ist ihr verfasser.

Allerdings erfährt sein ruhm dadurch keinen zuwachs, und mancher seiner verehrer hätte vielleicht gewüscht, dass

1 Interessant ist es, zu vergleichen, wie die nämliche stelle Bæda's von Aelfred und von Aelfric uibersetzt wird. Bæda: '- ut genti Anglorum aliquos ministros mitteret'. Aelfred: 'Joet he Angeldeode on Breotene onsende hwylce hugu lareowas'. Aelfric (Hom. II, s. 122): ' $\partial \propto t$ he Angelcynne sume lareowas asende'. 
Wright's ansicht die richtige wäre. Doch diese frage ist hier ganz nebensächlich. Denn in der wissenschaft handelt es sich um wahrheit und um weiter nichts als wahrheit.

\section{Excurs zu seite 453.}

Auch sonst lassen sich spuren des Griechischen im Angelsächsischen nachweisen. Die eigentliche volkssprache ist allerdings durch das Griechische nicht bereichert worden. Aber z. b. der angelsächsische urkundenstil enthält eine nicht unbedeutende \%ahl griechischer lehnwörter. Im 5. und 6. bande von Kemble's Codex Diplomaticus habe ich an 70 verschiedene griechische wörter ge\%:̈hlt, von denen einige, wie basileus, agius, cleronomus, cosmus, sophia, theus, häufig erscheinen. Ausserdem gibt es angelsächsische worte, welche direkte iibersetzungen aus dem Griechischen sind, und daher kaum vor 664 anzusetzen sind. Dazu ge-

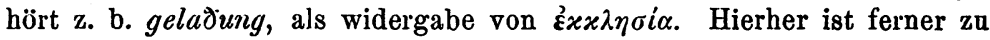
rechnen Hoeland, welches wort sich auch in den germanischen sprachen des kontinents findet. Auf welche weise wir uns Jesus durch heiland iibersetzt denken sollen, dariber sagt uns R. v. Raumer nichts (Einfluss des Christentums anf die althochd. Sprache, Stuttgart 1845, s. 355-356). Grimm's wörterbuch gibt heiland als uibersetzung von Salvator (Jesus). Doch ist dies schwerlich richtig. Fïr die ältere sprache würde neriand die richtige widergabe von Salvator = Jesus sein, wie denn Ulfilas in der tat nasjands und nicht hailjands hat. Der schlüssel zu dem richtigen verständniss von Hoeland $=$ heiland liegt, wie schon vor langer zeit von dem ersten herausgeber des Ormulum (zu v. 4270-i1) vermutet worden ist, in einer falschen etymologie des wortes Jesus. Da dies im neuen testamente vorkam, so sah man es als ein griechisches wort an

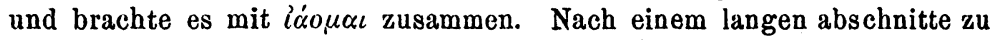
urteilen, den man in einem der evangelischen commentare Bæda's findet, iiber die allegorische bedeutung der sechs buchstaben des wortes 'I $\eta \sigma o \tilde{v}$, scheint der verfasser selbst an den griechischen ursprung des wortes geglaubt zu haben. Bestimmt wissen wir dies von Orm, denn er schreibt:

'- Jesuss o Grickisshe mal

onn Ennglissh iss Hælende'.

Natürlich hat Orm aus älteren quellen geschöpft. So würde also das wort 'heiland' nicht volkstiimlichen ursprungs sein, wie Vilmar annehmen will (Deutsche Altertïmer im Heliand als Einkleidung der evangelischen Geschichte. 2. ausg. Marburg 1862, s. 90), sondern einfach anf theologische gelehrsamkeit zuriickgehen. Wenn man nun die in England in der zweiten hälfte des 7. jahrhunderts vorhandene kenntniss des Griechischen beachtet, und ferner die tatsache, dass das nämliche wort sich in den kontinental-deutschen sprachen findet, so wird zum mindesten sehr wahrscheinlich, dass es von Angelsachsen zuerst gebildet worden ist. Es ist nicht wol denkbar, dass diese einen kirchlichen ausdruck Anglia, V. band. 
von den germanischen stämmen entliehen, welchen sie selbst erst christentum und kultur brachten. So wird man im allgemeinen die regel anfstellen können, dass bei übereinstimmung der übersetzten kirchlichen ausdrücke des Angelsächsischen und der kontinental-german. sprachen die priorität jenen zukommt. - Das wort munuclif = kloster scheint

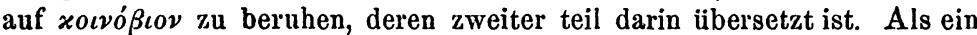
wahrscheinlich auf griechischen einflüssen beruhendes wort sei noch der ags. ausdruck für Aethiopier erwähnt, welcher in zwei formen vorkommt: Sizelhearwan und Sizelwaras. Der erste teil desselben kommt auch zusammengezogen vor: Sî̀. - Nicht nur poetisch kommt es vor, sondern

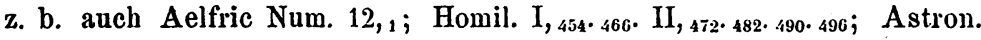
(bei Wright, Popul. Treat. on science dur. the m. a. s. 12); einleitung zum Neuen Testam. (bei Grein, 1. band der Denkm. ags. prosa s. 15); Aldh. Gloss. (Haupt's zeitschr. 18j3, s. 514). ${ }^{1}$ Wie der zweite teil von Sizelhearwa zu erklïren sei, vermag ich nicht anzugeben, ich finde auch nirgends eine vermutung darüber ausgesprochen. Ueber den ersten teil dagegen, $S i$ ¿ $e l=$ sonne, kann kein zweifel bestehen, und Sizelwaras bedeutet darnach ganz einfach sonnenmänner. Wie soll man sich diese ausdrucksweise erklären? Vielleicht geht sie zurück auf einen einfluss des bischofs Theodor, von dem Bæda sagt: 'Natus ex Tarso Ciliciae vir et saeculari et divina literatura Graece et Latine instructus'. Nach diesen worten darf man wol annehmen, dass Theodor die berühmten Aethiopica des Heliodor gelesen hatte, ein roman, der sich seit seinem erscheinen bis an das ende des mittelalters einer ausserordentlichen beliebtheit erfreute (vgl. Rohde, Der griech. Roman und seine Vorläufer, Leipzig 1876, s. 522). In diesem romane werden die Aethioper in engen

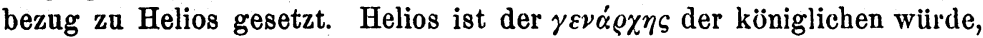
Helios ist der beschiitzer des landes, Helios ist der gott, den sie besonders anbeten. Es lässt sich denken, dass Theodor seinen schülern von diesem berühmten romane erzählte, und dass ihre einbildungskraft sich damit lebhaft beschäftigte. Durch den roman angeregt, nannten sie die Aethiopier sonnenleute und verschafften durch ihren einfluss diesem worte allgemeine geltung.

LEIPZIG.

K. A. Maktin Hartanan.

1 Auch in der ags. iibertragung der schrift: De Rebus in Orienle mirabilibus c. XXXIII findet sich silhearnan. Spelmann's Psalt. it, 34 silhearwena, syzilhearwan. 71, 9 sylhearwena, sizylhearwan, sielhearwan u. s. Aelfr. Gen. 2, 13 silhearwena.

R. W. 